VERSION OCTOBER 2, 2018: FM

Preprint typeset using LTEX style emulateapj v. 11/10/09

\title{
REFINING THE ASSOCIATIONS OF THE Fermi LARGE AREA TELESCOPE SOURCE CATALOGS
}

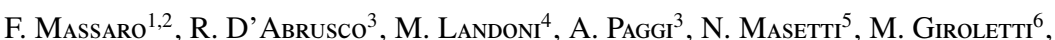 \\ H. Otí-Floranes ${ }^{7,8}$, V. Chavushyan ${ }^{9}$, E. Jiménez-Bailón ${ }^{7}$, V. Patiño-Álvarez ${ }^{9}$, S. W. Digel ${ }^{10}$, Howard A. Smith ${ }^{3} \&_{\text {G. Tosti }}^{11}$ \\ version October 2, 2018: fm
}

\section{ABSTRACT}

The Fermi-Large Area Telescope (LAT) First Source Catalog (1FGL) was released in February 2010 and the Fermi-LAT 2-Year Source Catalog (2FGL) appeared in April 2012, based on data from 24 months of operation. Since their releases, many follow up observations of unidentified gamma-ray sources (UGSs) were performed and new procedures to associate gamma-ray sources with potential counterparts at other wavelengths were developed. Here we review and characterize all the associations as published in the 1FGL and 2FGL catalog on the basis of multifrequency archival observations. In particular we located 177 spectra for the low-energy counterparts that were not listed in the previous Fermi catalogs, and in addition we present new spectroscopic observations of $8 \gamma$-ray blazar candidates. Based on our investigations, we introduce a new counterpart category of "candidate associations" and propose a refined classification for the candidate low-energy counterparts of the Fermi sources. We compare the 1FGL-assigned counterparts with those listed in the 2FGL to determine which unassociated sources became associated in later releases of the Fermi catalogs. We also search for potential counterparts to all the remaining unassociated Fermisources. Finally, we prepare a refined and merged list of all the associations of the 1FGL plus 2FGL catalogs that includes 2219 unique Fermi objects. This is the most comprehensive and systematic study of all the associations collected for the $\gamma$-ray sources available to date. We conclude that $80 \%$ of the Fermi sources have at least one known plausible gamma-ray emitter within their positional uncertainty regions.

Subject headings: methods: statistical - galaxies: active - quasars: general - surveys

\section{INTRODUCTION}

Despite the large progress in $\gamma$-ray source localization made by the Fermi Large Area Telescope (LAT) (Atwood et al. 2009) coupled with the improvements in our knowledge of the diffuse Galactic $\gamma$-ray emission (e.g., Moskalenko et al. 2007; Abdo et al. 2009), the positional uncertainties of the Fermi sources are still large with respect to typical localization precisions at X-ray and lower energies, making a significant fraction of the sources in the $\gamma$ ray sky yet unknown (Abdo et al. 2010a; Nolan et al. 2012). About $43 \%$ of the Fermi sources detected in the FermiLarge Area Telescope (LAT) First Source Catalog (1FGL Abdo et al. 2010a) were listed as UGSs while there were $\sim 33 \% \gamma$-ray objects unassociated in the Fermi-LAT 2-Year

\footnotetext{
${ }^{1}$ Dipartimento di Fisica, Università degli Studi di Torino, via Pietro Giuria 1, I-10125 Torino, Italy

${ }^{2}$ Yale Center for Astronomy and Astrophysics, Physics Department Yale University, PO Box 208120, New Haven, CT 06520-8120, USA

${ }^{3}$ Harvard - Smithsonian Astrophysical Observatory, 60 Garden Street, Cambridge, MA 02138, USA

${ }^{4}$ INAF-Osservatorio Astronomico di Brera, Via Emilio Bianchi 46, I23807 Merate, Italy

${ }^{5}$ INAF - Istituto di Astrofisica Spaziale e Fisica Cosmica di Bologna, via Gobetti 101, 40129, Bologna, Italy

${ }^{6}$ INAF Istituto di Radioastronomia, via Gobetti 101, 40129, Bologna, Italy

${ }^{7}$ Instituto de Astronomía, Universidad Nacional Autónoma de México, Apdo. Postal 877, Ensenada, 22800 Baja California, México

${ }^{8}$ Centro de Radioastronomía and Astrofísica, UNAM, Campus Morelia, México

${ }^{9}$ Instituto Nacional de Astrofísica, Óptica y Electrónica, Apartado Postal 51-216, 72000 Puebla, México

${ }^{10}$ SLAC National Accelerator Laboratory and Kavli Institute for Particle Astrophysics and Cosmology, 2575 Sand Hill Road, Menlo Park, CA 94025, USA

${ }^{11}$ Dipartimento di Fisica, Università degli Studi di Perugia, 06123 Perugia, Italy
}

Source Catalog (2FGL Nolan et al. 2012).

To decrease the number of unidentified gamma-ray sources (UGSs) many methods based on multifrequency approaches or statistical analyses have been recently adopted. Radio follow up observations of the Fermi UGSs have already been performed (e.g., Kovalev 2009; Hovatta et al. 2012; Petrov et al. 2013; Hovatta et al. 2014; Schinzel et al. 2014) and the Swift X-ray survey for all the UGSs listed in the Fermi source catalogs is still on going ${ }^{12}$ (e.g., $\quad$ Mirabal \& Halpern 2009; $\quad$ Paggi et al. 2013; Takeuchi et al. 2013; Stroh et al. 2013; Acero et al. 2013). Additional X-ray observations performed with Chandra and Suzaku improved our knowledge on the UGSs (e.g., Maeda et al. 2011; Cheung et al. 2012; Kataoka et al. 2012; Takahashi et al. 2012). Statistical studies based on the $\gamma$-ray source properties also allowed us to recognize the nature of the potential counterparts for the UGSs (e.g., Ackermann et al. 2012; Mirabal et al. 2012; Hassan et al. 2013; Doert \& Errando 2014). Moreover a tight connection between the infrared (IR) sky seen by Wide-Field Infrared Survey Explorer (WISE Wright et al. 2010) and the Fermi one has been recently discovered for blazars, the rarest class of active galaxies (Massaro et al. 2011a; D'Abrusco et al. 2012; D'Abrusco et al. 2013). These works greatly decreased the fraction of UGSs with no assigned counterpart at low energies (Massaro et al. 2012a; Massaro et al. 2012b; Massaro et al. 2013a). More recently also low frequency radio observations (i.e., below $\sim 1 \mathrm{GHz}$ ) revealed a new spectral behavior that allowed us to search for blazar-like sources lying within the positional uncertainty regions of the UGSs (Massaro et al. 2013b; Nori et al. 2014). Additional

\footnotetext{
12 http://www.swift.psu.edu/unassociated/
} 
studies have been also carried out with near-infrared observations (Raitieri et al. 2014) as well as in the sub millimeter range (e.g., Giommi et al. 2012; López-Caniego et al. 2013). Optical spectroscopic campaigns also have been crucial to disentangle and/or confirm the natures of the low-energy counterparts selected with different methods (e.g., Masetti et al. 2013; Shaw et al. 2013a; Shaw et al. 2013b; Paggi et al. 2014; Massaro et al. 2014b; Landoni et al. 2014).

The main aim of the analysis presented here is to confirm the current status of the associations for the 1FGL and the 2FGL $\gamma$-ray sources. Thus we prepare a single list, created by merging the unique sources in the 1FGL and in the 2FGL catalogs. On the basis of the information reported in the First Fermi-LAT Catalog of Sources Above $10 \mathrm{GeV}$ (1FHL Ackermann et al. 2013) and in both the First and the Second Catalog of Active Galactic Nuclei Detected by Fermi (1LAC and 2LAC, respectively Abdo et al. 2010b; Ackermann et al. 2011a), together with an extensive literature search in multifrequency archives, we verify the current status of the $\gamma$-ray associations. In addition, we also present new optical spectroscopic observations of eight $\gamma$-ray blazar candidates selected during our multifrequency study. The result is a refined and merged list of all the associations for the 1FGL-plus-2FGL catalog with updated information on the low-energy counterparts including specific multifrequency notes.

The paper is organized as follows: in Section 2 we discuss the Fermi procedures to assign identifications and associations with potential counterparts at low energies, and the categories of $\gamma$-ray source associations. Section 3 describes the content and the main properties of both the 1FGL and the 2FGL catalogs. In Section 4 we introduce an updated $\gamma$-ray source classification for the counterparts of the Fermi detected objects. Details on the correlation with multifrequency databases and catalogs are in Section 5. In Section 6 we present the refined version of the associations listed in the Fermi catalogs (hereinafter designated 1FGLR and 2FGLR, respectively, for brevity). The analysis of the infrared colors for the sources associated in the 1FGL is given in Section 7 and new optical spectroscopic observations of $\gamma$-ray blazar candidates are presented in Section 8. In Section 9 we compare our results with those achieved from statistical analyses performed on the UGSs listed in both the 1FGL and the 2FGL. In Section 10 we speculate on both the radio- $\gamma$-ray and the IR- $\gamma$-ray connections for the Fermi blazars to check the consistency of sources classified as blazar candidates with these multifrequency behaviors. The summary and conclusions are given in Section 11. We use cgs units unless stated otherwise. Spectral indices, $\alpha$, are defined by flux density, $\mathrm{S}_{v} \propto v^{-\alpha}$ and WISE magnitudes at [3.4], [4.6], [12], [22] $\mu \mathrm{m}$ (i.e., the nominal bands) are in the Vega system. For numerical results a flat cosmology was assumed with $H_{0}=67.3 \mathrm{~km} \mathrm{~s}^{-1} \mathrm{Mpc}^{-1}$, $\Omega_{M}=0.315$ and $\Omega_{\Lambda}=0.685$ (Planck collaboration 2014). The astronomical survey and source class acronyms used in the paper are listed in Table 1.

\section{CATEGORIES OF GAMMA-RAY SOURCE ASSOCIATIONS}

In all the Fermi catalogs there is an important distinction between identification of low-energy counterparts for the Fermi sources and association. Identification is based on i) spin or orbital periodicity (e.g., pulsars, binary systems) or on ii) correlated variability at other wavelengths (e.g., blazars, active galaxies) or on iii) the con-
TABLE 1

LIST OF ACRONYMS USED IN THE PAPER.

\begin{tabular}{|c|c|}
\hline Acronym & Term \\
\hline 1FGL & Fermi-Large Area Telescope First Source Catalog \\
\hline 1FGLR & 1FGL Refined association catalog \\
\hline $1 \mathrm{FHL}$ & First Fermi-Large Area Telescope Catalog of Sources Above $10 \mathrm{GeV}$ \\
\hline $1 \mathrm{LAC}$ & First Catalog of Active Galactic Nuclei Detected by Fermi \\
\hline 1XSPS & Deep Swift X-Ray Telescope Point Source Catalog \\
\hline 2FGL & Fermi-Large Area Telescope 2-Year Source Catalog \\
\hline 2FGLR & 2FGL Refined association catalog \\
\hline $2 \mathrm{LAC}$ & Second Catalog of Active Galactic Nuclei Detected by Fermi \\
\hline 2MASS & Two Micron All-Sky Survey \\
\hline $3 \mathrm{C}$ & Third Cambridge Catalogue of Radio Sources \\
\hline 6dFGS & Six-degree-Field Galaxy Redshift Survey \\
\hline AGN & Active Galactic Nucleus \\
\hline AGU & AGN of Uncertain type \\
\hline AT20G & Australia Telescope $20 \mathrm{GHz}$ Survey \\
\hline ATNF & Australia Telescope National Facility \\
\hline BZB & BL Lac object \\
\hline BZQ & flat spectrum radio quasar \\
\hline CRATES & Combined Radio All-Sky Targeted Eight-GHz Survey radio catalog \\
\hline $\mathrm{CSC}$ & Chandra Source Catalog \\
\hline СT & Classification Tree method \\
\hline FIRST & VLA Faint Images of the Radio Sky at Twenty-Centimeters \\
\hline GB6 & Green Bank 6-cm Radio Source Catalog \\
\hline GLC & Globular Cluster \\
\hline HMB & High Mass X-ray Binary \\
\hline $\mathrm{KDE}$ & Kernel Density Estimation \\
\hline IRAF & Image Reduction and Analysis Facility \\
\hline IRAS & InfraRed Astronomical Satellite \\
\hline LAT & Large Area Telescope \\
\hline LBA & Australian Long Baseline Array \\
\hline LCS1 & LBA Calibrator Survey \\
\hline LORCAT & Low-frequency Radio Catalog of flat-spectrum Sources \\
\hline LR & Logistic Regression method \\
\hline MGPS & Molonglo Galactic Plane Survey \\
\hline MSP & Millisecond Pulsar \\
\hline NED & NASA/IPAC Extragalactic Database \\
\hline NOV & Nova \\
\hline NRAO & National Radio Astronomy Observatory \\
\hline NVSS & NRAO VLA Sky Survey Catalog \\
\hline OAGH & Observatorio Astrofisico Guillermo Haro \\
\hline OAN & Observatorio Astronómico Nacional \\
\hline PKS & Parkes Southern Radio Source catalog \\
\hline PMN & Parkes-MIT-NRAO Surveys \\
\hline PSR & Pulsar \\
\hline PWN & Pulsar wind nebula \\
\hline RBSC & ROSAT Bright Source Catalog \\
\hline RFSC & ROSAT Faint Source Catalog \\
\hline SDSS & Sloan Digitized Sky Survey \\
\hline SED & Spectral Energy Distribution \\
\hline SFR & Star Forming - HII regions \\
\hline SNR & Supernova Remnant \\
\hline SUMSS & Sydney University Molonglo Sky Survey \\
\hline TEXAS & Texas Survey of Radio Sources at $365 \mathrm{MHz}$ \\
\hline UGS & Unidentified Gamma-ray Source \\
\hline VLA & Very Large Array \\
\hline VLSS & VLA Low-Frequency Sky Survey Discrete Source Catalog \\
\hline USNO & United States Naval Observatory \\
\hline XMMSL & XMM-Newton Slew Survey \\
\hline WENSS & Westerbork Northern Sky Survey \\
\hline WISE & Wide-Field Infrared Survey Explorer \\
\hline WISH & Westerbork in the Southern Hemisphere Source Catalog \\
\hline WSRT & Westerbork Synthesis Radio Telescope \\
\hline WSRTGP & WSRT Galactic Plane Compact 327-MHz Source Catalog \\
\hline
\end{tabular}

sistency between the measured angular sizes in $\gamma$-ray and at lower energies (e.g., supernova remnants). On the other hand, the association designation indeed depends on the results of different procedures adopted in both Fermi catalogs (Abdo et al. 2010a; Nolan et al. 2012). These procedures are:

1. The Bayesian Association Method: Initially applied to associate EGRET sources with flat-spectrum radio sources (e.g., Mattox et al. 1997; Mattox et al. 2001; Abdo et al. 2010a), this method assesses the probability of association between a $\gamma$-ray source and a candidate counterpart taking into account their local densities. Local density is estimated simply by counting 
candidates in a nearby region of the sky.

2. The Likelihood Ratio method: Used to search for possible counterparts in uniform surveys in the radio and in $\mathrm{X}$-ray bands, this procedure was originally proposed by Richter (1975) and subsequently applied by and modified by de Ruiter, Willis \& Arp (1977), Prestage \& Peacock (1983), Wolstencroft et al. (1986) and by Sutherland \& Saunders (1992).

3. The $\log N-\log S$ association method: This is a modified version of the Bayesian method for blazars taking into account their $\log N-\log S$ (see Abdo et al. 2010b; Ackermann et al. 2011a, for more details).

There are several differences between the source associations presented between the 1FGL and the 2FGL catalogs mostly related to the $\gamma$-ray analysis and to the improvements achieved in the models of Galactic $\gamma$-ray diffuse emission as well as in evaluation of the LAT response functions. Improving the Fermi source localization not only makes easier the search for low-energy counterparts but allows more accurate estimates of the association probability since they depend on the $\gamma$-ray positional uncertainty (see e.g., Abdo et al. 2010b; Ackermann et al. 2011a). These differences also relate to updates of comparison surveys and catalogs used to search for the low-energy counterparts and on multifrequency observations performed on UGS samples.

The use of catalogs/surveys that were previously not considered for the Fermi catalog preparation, as for example the infrared all-sky survey performed by WISE and the lowfrequency radio catalogs, revealing new connections between the low and the high-energy skies (e.g., Massaro et al. 2011a; D'Abrusco et al. 2013; Massaro et al. 2013b) has also decreased the unknown fraction of the $\gamma$-ray sky (e.g., Massaro et al. 2012b; Massaro et al. 2013a). This affects the Fermi associations because the methods adopted to assign low-energy counterparts depend on the source densities of the catalogs (Nolan et al. 2012; Ackermann et al. 2011a). A better estimate of the counterpart density leads to a better estimate of the false positive associations and of the association probability and this implies that a previously unassociated source could have an assigned counterpart in a new release of the Fermi catalog.

Motivated by the changes occurring between the 1FGL and the 2FGL associations here we propose to introduce, together with identified and associated sources, the category of "candidate associations". These are $\gamma$-ray sources that have a potential low-energy counterpart in a specific class of wellknown $\gamma$-ray emitters lying within the Fermi positional uncertainty region at $95 \%$ level of confidence and/or having angular separations between the Fermi and the counterpart position smaller than the maximum one for all the associated sources of the same class. Candidate associations have the potential to be promoted to associations in the future (see Section 4).

We considered as counterpart classes for the candidate associations only a few kinds of sources: i) blazar-like sources, as defined in the following, ii) known pulsars (PSRs), iii) pulsar wind nebulae (PWNe), supernova remnants (SNRs) and star forming and HII regions (hereinafter simply indicated as SFRs). The first two source classes constitute the two largest populations of known $\gamma$-ray emitters while the other choices were considered because 15 out of the 44 SNRs and 10 out of the 63 PSRs and PWNe associated in the 1FGL lie within SFRs and a similar situation occurs for the 2FGL.
Our choice concerning the SFRs, especially for the most massive cases, is indeed motivated by the possibility that they could host type $\mathrm{O}$ and/or B stars, potentially associated with open star clusters, and this being relatively likely to have some members that have progressed to become a PSR, PWN, and/or SNR, all known classes of $\gamma$-ray sources (see also Montmerle 1979; Montmerle 2009).

In addition, considering SFRs as candidate associations is also motivated by the recent idea, partially supported by the Fermi discovery of $\gamma$-ray emission arising from Eta Carinae (Abdo et al. 2010c), that shells detected in the radio, infrared, and X-rays could mark termination shocks of stellar winds. These winds interacting with the stars' natal molecular clouds can be considered as plausible sites to accelerate particles that emit $\gamma$-rays (e.g., Araudo et al. 2007; Bosch-Ramon et al. 201; Rowell et al. 2010). Bowshocks are associated with many source classes such as PSRs, cataclysmic variables, colliding wind binaries, cometary $\mathrm{H}$ II regions and particles accelerated therein can radiate up to the MeV-GeV energy range (see e.g. Del Valle \& Romero 2012). However we remark that evidence of $\gamma$-ray emission from colliding wind binaries associated with Wolf-Rayet stars was not found by Fermi, for a selected sample of 7 sources (Werner et al. 2013); although, as highlighted by the same authors, searching for such $\gamma$-ray detections is extremely complicated due to the uncertainties of the diffuse emission in the Galactic plane.

Some candidate associations lack multifrequency information as well as correct spectroscopic identifications but exhibit some characteristic features, typical of known $\gamma$ ray emitters, mainly blazars and PSRs. They were not associated in the previous releases of the Fermi catalogs mostly because they were not listed in one of the appropriate comparison catalogs of potential counterparts used to associated Fermi sources. However once follow up observations were performed (see e.g. Paggi et al. 2013; Masetti et al. 2013; Petrov et al. 2013; Massaro et al. 2014b; Landoni et al. 2014, for blazar-like candidates), then they could be promoted to associated sources in new releases of the Fermi catalogs. In addition, within this category we also considered Fermi sources having SFRs within their $\gamma$-ray positional uncertainty regions for which multifrequency observations could reveal the presence of unknown PSRs, PWNe and SNRs, already counterparts of $\sim 5 \%$ of the $\gamma$-ray sources.

We emphasize three advantages obtained from introducing the concept of candidate associations:

1. Designating candidate association category allows us to flag potential counterparts that might be associated or identified in future releases of the Fermi catalogs, as previously occurred to 171 1FGL sources then recognized, classified and associated in the 2FGL. This also facilitates planning follow up multifrequency observations, in particular spectroscopic ones, necessary to determine and/or confirm their nature.

2. The category of candidate associations permits us to establish which is the number of Fermi sources not yet having potential counterparts. The precise knowledge of this number is extremely relevant to set better constraints on dark matter scenarios (e.g., Zechlin et al. 2012; Mirabal et al. 2012; Mirabal 2013a; Mirabal 2013b; Berlin \& Hooper 2014). 
3. For all the methods adopted to associate sources in the Fermi catalogs, the resulting associations depend on the comparison catalog/survey used. The same low-energy counterpart may be associated with a Fermi source using a certain catalog but not with a different survey, even when the positions in the two catalogs are the same. This occurs because the source density of the comparison catalogs, which is used to determine the probability of false positive associations, affects the association probability. Thus observing these candidate associations with follow up campaigns to determine their natures will permit us to add blazars and pulsars to the correct comparison catalogs and associate them in future releases of the Fermi surveys.

To reiterate the importance of having candidate associations we highlight the recent work of Schinzel et al. (2014) on radio very-long-baseline interferometry (VLBI) observations of Fermi unassociated sources in the 2FGL (see also Petrov et al. 2013). In the final list of high-confidence associations presented in Schinzel et al. (2014) more than $90 \%$ of the sources are also detected in the major radio surveys such as the National Radio Astronomy Observatory (NRAO) Very Large Array (VLA) Sky Survey Catalog (NVSS; Condon et al. 1998) and the Sydney University Molonglo Sky Survey (SUMSS; Mauch et al. 2003). These counterparts were not associated using the methods adopted in Fermi catalogs due to the high density of sources at low flux level in the above surveys. However thanks to the high resolution radio follow up observations, which reveled the presence of radio compact cores, it has been possible to use a different selected sample of potential counterparts to find new associations via the likelihood procedure (Schinzel et al. 2014; Petrov et al. 2013). Thus it is extremely important to have targets for follow up observations that, once performed, will allow us to insert the potential counterparts in the correct catalogs used to search $\gamma$-ray associations.

Identifications, associations and candidate associations' of $\gamma$-ray sources are labelled in both the refined version of the Fermi catalogs as the category of the $\gamma$-ray source association. These are defined as: identified (I), associated (A), candidate association (C) in the tables while sources for which no potential counterpart was found are assigned to the UGS category $(\mathrm{U})$.

\section{THE Fermi CATALOGS}

\subsection{The Fermi-Large Area Telescope First Source Catalog}

The 1FGL catalog ${ }^{13}$ contains 1451 sources detected and characterized in the $100 \mathrm{MeV}$ to $100 \mathrm{GeV}$ energy range. For each Fermi source listed therein, positional uncertainty regions at $68 \%$ and $95 \%$ level of confidence, results from spectral fits as well as flux measurements in five energy bands are reported (Abdo et al. 2010a). Firm identification or plausible association with a low-energy counterpart was provided for each 1FGL source on the basis of the comparison with other astronomical catalogs and on the basis of the association procedures previously mentioned (see Section 2 and Abdo et al. 2010a for details).

The published 1FGL catalog lists several classes of counterparts: 63 pulsars (PSRs), 7 associated plus 56 identified (24 of them discovered thanks to the Fermi-LAT observations), 2 pulsars wind nebulae (PWNe) $44 \gamma$-ray sources cor-

${ }^{13}$ http://fermi.gsfc.nasa.gov/ssc/data/access/lat/1yr_catalog/ responding to 38 SNRs, 41 associated and 3 identified, 8 globular clusters (GLC), 2 high mass X-ray binaries (HMB), all identified, 295 BL Lac objects (BZBs) 278 flat spectrum radio quasars (BZQs), 28 non blazar-like active galactic nuclei (AGNs) 92 AGN of uncertain type (AGUs) 6 normal galaxies and 2 starburst galaxies. The remaining 630 Fermi sources are all UGSs.

A detailed comparison between the 1FGL and the 2FGL catalog is reported in Nolan et al. (2012). There are 1099 sources included in both Fermi catalogs with only 381 1FGL sources being listed in the 1FHL (Ackermann et al. 2013). It is worth noting that 267 out of 1099 sources in common between 1FGL and 2FGL changed classification from 1FGL to $2 \mathrm{FGL}$ and among them 172 , previously unassociated in 1FGL, obtained an assigned low-energy counterpart in 2FGL. This sample of modified associations includes: 16 sources indicated as generic AGNs that have been classified plus one that became a UGS; 54 AGUs that have been recognized as blazars (either BZBs or BZQs) and 2 as AGNs; then 17 blazars actually changed their classification from 1FGL to 2FGL thanks to new spectroscopic data available and, among them, 6 actually disappeared since they were listed in the 2FGL as unassociated as occurred for other 5 sources of Galactic origin (i.e., PSRs, PWNe and SNRs). On the other hand, there are 172 UGSs of the 1FGL that appear to be associated in the 2FGL: 20 associated with PSRs, 11 with SNRs and 1 with a GLC while all the others with various AGN classes (i.e. blazars, radio galaxies, Seyfert galaxies etc.).

The 1FGL catalog includes 216 sources with $\gamma$-ray analysis flags and their detection has to be considered carefully since they could have been artifacts of the analysis (Abdo et al. 2010a). In particular, 103 of these 216 1FGL flagged sources have the "c" flag following the 1FGL name; this indicates that the source is found in a region with bright and/or possibly incorrectly modeled diffuse emission. On the other hand any non-zero entry in the Flags column indicates inconsistencies found during the analysis (see Abdo et al. 2010a, for details). However we note that 36 out of 103 "c"-flagged 1FGL sources are also detected in the 2FGL and only 25 of them were still indicated with a "c" flag therein. Thus we decided to keep all the c-flagged sources in our analysis since they could be useful for reference in future releases of Fermi catalogs.

\subsection{The Fermi-Large Area Telescope Second Source Catalog}

The 2FGL catalog ${ }^{14}$ based on 24 months of Fermi operation lists $1873 \gamma$-ray sources (Nolan et al. 2012). It includes:

- 108 PSRs, 25 associated plus 83 identified;

- 3 PWNe, all 3 identified;

- $61 \gamma$-ray sources corresponding to 50 SNRs, 55 associated plus 6 identified;

- 11 associated globular clusters (GLC), 4 high mass Xray binaries (HMB), all identified and 1 nova (NOV),

among the Galactic sources, while in the extragalactic sky:

- 435 BL Lac objects (BZBs), only 7 identified;

- 370 flat spectrum radio quasars (BZQs), 17 identified;

${ }^{14}$ http://fermi.gsfc.nasa.gov/ssc/data/access/lat/2yr_catalog/ 
- 11 non blazar-like active galactic nuclei (AGNs), 1 identified;

- 257 AGNs of uncertain type (AGUs);

- 6 normal galaxies and 4 starburst galaxies .

The remaining 577 Fermi sources were are all UGSs.

The 2FGL catalog lists 472 sources with $\gamma$-ray analysis flags and 155 of them also have the "c" flag following the 2FGL name. It is also worth noting that with respect to the 1FGL catalog the fraction of UGSs is decreased from 630 out of 1451 sources $(\sim 43 \%)$ to 577 out of $1873(\sim 31 \%)$ in the 2FGL.

\section{INTRODUCING THE CLASSIFICATION FOR THE LOW-ENERGY COUNTERPARTS OF THE Fermi SOURCES}

We adopt the following class definitions for Galactic counterparts of the Fermi sources, summarized in Table 2: high mass X-ray binary (hmb), globular cluster (glc), nova (nov), millisecond pulsar (msp), pulsar (psr), pulsar wind nebula (pwn), supernova remnant (snr), star forming region or $\mathrm{H}$ II region (sfr) and binary star (bin). The classes that we assign for extragalactic counterparts are BL Lac object (bzb), quasar radio loud of flat spectrum type (bzq), blazar of uncertain type (bzu), radio galaxy (rdg), normal galaxy (gal), starburst galaxy (sbg) and Seyfert galaxy (sey). We note that we used the three-letter designators to indicate the class for each counterpart; these are shown in lower case to differentiate them from the acronyms used throughout the paper (see Table 1). We emphasize that the labels used for the extragalactic blazar subclasses are used only if the counterpart corresponds to a source classified in the Roma-BZCAT (Massaro et al. 2009; Massaro et al. 2011b). We used version 4.1 of the Roma-BZCAT in our analysis. We also emphasize that the proposed classification depends on the availability of the optical spectroscopic information for the lowenergy counterparts.

We also considered as a counterpart class blazar candidates (bcn) to indicate sources having at least one of the following requirements: 1) being classified as BL Lac candidate in the Roma-BZCAT; 2) sources that belong to the Combined Radio All-Sky Targeted Eight-GHz Survey radio catalog (CRATES; Healey et al. 2007) with a BL Lac-like or a quasar-like optical spectrum available in literature; 3 ) radio sources with a blazar-like optical spectrum available in literature (i.e., a published spectrum or a description of the features found in the near infrared-optical band) and with a WISE counterpart.

Finally, we labelled as unclassified counterparts (unc) those sources satisfying at least one of the following criteria: 1) WISE Infrared colors similar to those of $\gamma$-ray blazars selected according to the association methods developed by D'Abrusco et al. (2013) and/or with the kernel density estimator analysis (Massaro et al. 2012a; Massaro et al. 2012c, see also Section 7 for a refined analysis); 2) a flat radio spectrum at frequencies below $\sim 1 \mathrm{GHz}$ (i.e., $\alpha<0.5$ ); 3) a radio source with or without an X-ray counterpart associated with the methods adopted in the 1LAC and the 2LAC; 4) a radio and/or an X-ray source that lies at angular separation between the Fermi and the counterpart positions smaller than the maximum one for the blazar class (i.e., $0.4525^{\circ}$ corresponding to 2FGL J1746.0+2316). All the remaining sources are still unidentified (ugs).

The proposed classification scheme has three main advantages.
1. To have a homogeneous classification of the blazars listed in our analysis and to avoid misclassifications we adopted the following criterion. We indicate as blazars only sources that were previously recognized and classified in the Roma-BZCAT. There are indeed several counterparts classified as $\mathrm{BZB}$ or $\mathrm{BZQ}$ listed in the $1 \mathrm{LAC}$ and in the $2 \mathrm{LAC}$ catalogs that do not have the same classification according to the Roma-BZCAT or for which no optical spectra were found in literature to confirm their classification.

2. We do not classify a source with a radio and/or X-ray counterpart as an active galaxy of uncertain type (AGU) since these requirements are not strictly sufficient to claim a source as an AGN or as blazar-like and the lack of multifrequency information does not allow us to determine precisely its nature. Consequently, we introduced the ben and the unc classes. Once optical spectra and multifrequency observations become available a refined classification will be provided.

3. We introduced the sfr class for cases where Fermi position is consistent with a known massive SFR for three reasons. First, to highlight the possibility that an unknown SNR, PWN and/or PSR is embedded therein, for which multifrequency observations are necessary to confirm their presence. Second, because the use of this information could be adopted to refine $\gamma$-ray diffuse models in future releases of the Fermi catalogs. In particular, the largest fraction of these SFR potential associations were determined using the recent WISE Catalog of Galactic H II Regions ${ }^{15}$ (Anderson et al. 2014), which was not available during the preparation of the currently-published Fermi catalogs. This could also reveal new correspondences between the uncertainties of the diffuse $\gamma$-ray background models and regions of interstellar medium in the Galactic plane. Third, this could be useful for future investigations of the environments of the Galactic $\gamma$-ray sources.

Finally, we remark that for the sources indicated as candidate associations and classified as SFRs, SNRs, PWNs and PSRs we investigated the radio and infrared images available in the NVSS, the Westerbork Synthesis Radio Telescope (WSRT) Galactic Plane Compact 327-MHz Source Catalog (WSRTGP; Taylor et al. 1996), the Molonglo Galactic Plane Survey ${ }^{16}$ (MGPS; Green et al. 1999) catalogs and in the WISE archives searching for signatures of potential $\gamma$-ray emitters.

In Figures 1 and 2 we show two cases of $\gamma$-ray sources having extended structures associated with an open star cluster in a SFR found within their Fermi positional uncertainty regions. These are two examples of star clusters where some members could have been progressed becoming a PSR, PWN, and/or a SNR, and so being a plausible counterpart for the $\gamma$-ray emission.

Then in Figures 3 and 4 we report the best two cases of SFRs for which a signature of a shell-like or ring-like extended structures was found within the Fermi positional uncertainty region that could be ascribed as a shock signature potentially responsible for the $\gamma$-ray emission.

\footnotetext{
15 http://astro.phys.wvu.edu/wise/

16 http://www.astrop.physics.usyd.edu.au/MGPS/
} 

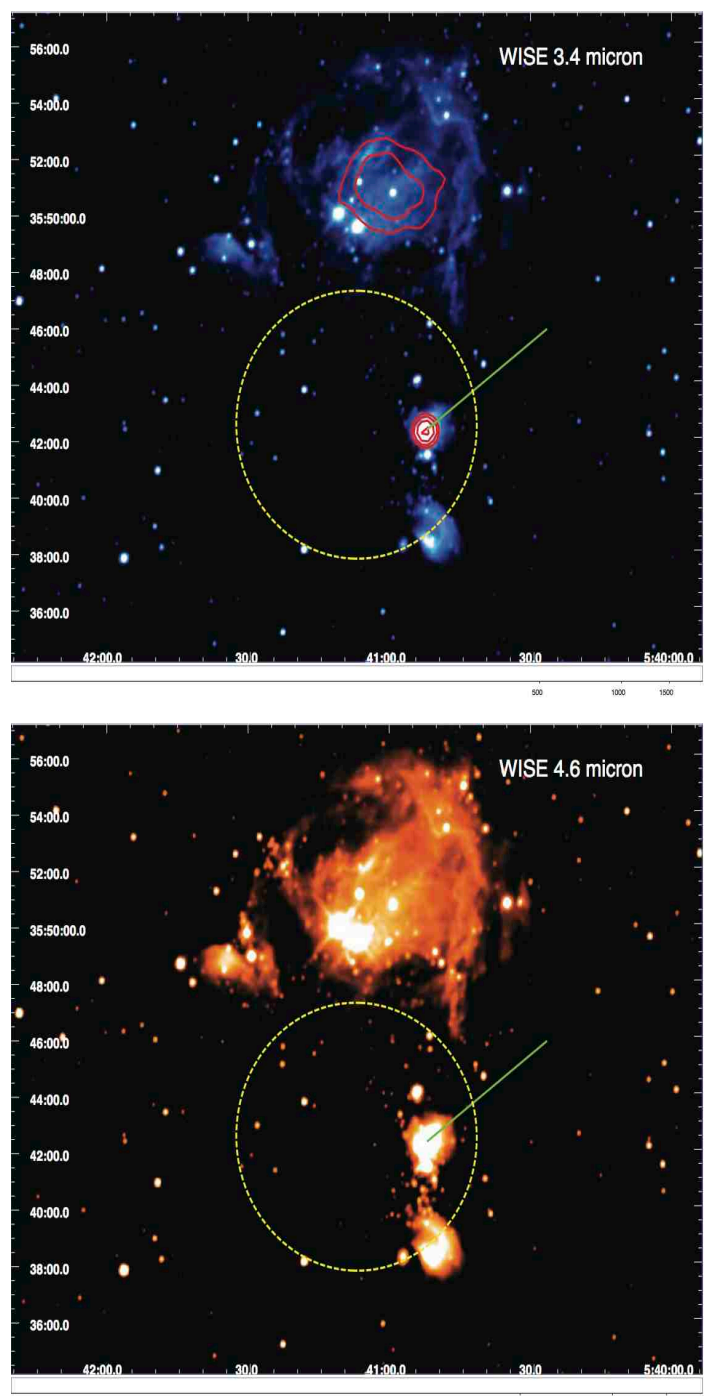

FIG. 1.- The WISE images of the Fermi source 1FGLJ0541.1+3542c in two different bands at $3.4 \mu \mathrm{m}$ (top), $4.6 \mu \mathrm{m}$ (bottom). In each image the yellow ellipse corresponds to the Fermi positional uncertainty region at $68 \%$ level of confidence. The red contours on the WISE image at $3.4 \mu \mathrm{m}$ indicate the radio brightness at $1.4 \mathrm{GHz}$ from the NVSS. The radio-infrared extended structure, marked by the green arrow, suggests that a shock occurred in the interstellar medium that could be responsible for the $\gamma$-ray emission. The radio source is also detected in the Westerbork Northern Sky Survey (WENSS; Rengelink et al. 1997) and it is associated with an open star cluster.

\section{CORRELATION WITH EXISTING DATABASES}

We searched the following major radio, infrared, optical and X-ray surveys and both the NASA Extragalactic Database (NED) ${ }^{17}$ and the SIMBAD Astronomical Database ${ }^{18}$ to verify whether multifrequency information can confirm the natures of uncertain counterparts and blazar candidates. In the following we list all the catalogs and the surveys searched for our investigation; the abbreviations correspond to the designators used for the multifrequency notes in Table 4.

Below $\sim 1 \mathrm{GHz}$ radio frequency we searched the VLA Low-Frequency Sky Survey Discrete Source Catalog (VLSS; Cohen et al. 2007, - V) and the recent revision VLLSr ${ }^{19}$

\footnotetext{
${ }^{17}$ http://ned.ipac.caltech.edu/

18 http://simbad.u-strasbg.fr/simbad/

19 http://heasarc.gsfc.nasa.gov/W3Browse/all/vlssr.html
}
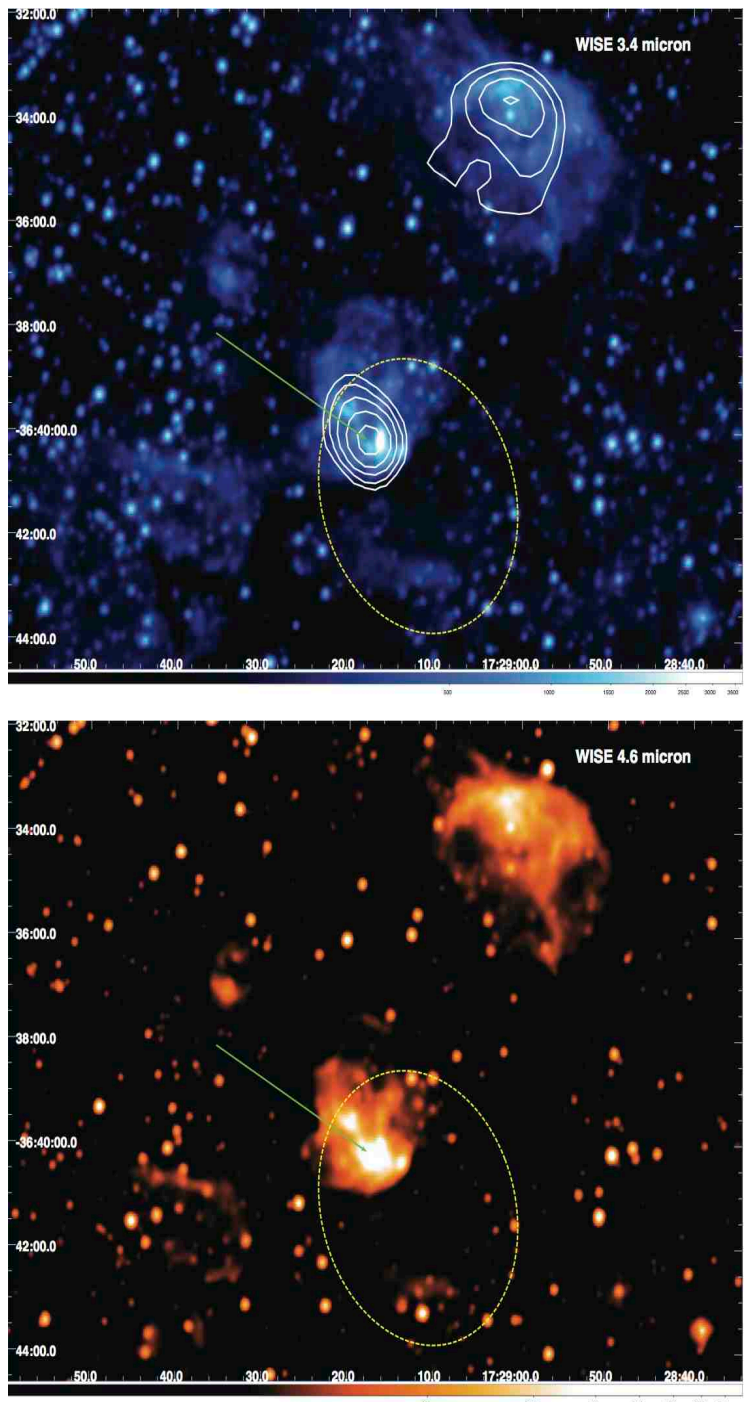

FIG. 2.- The WISE images of the Fermi source 1FGLJ1729.1-3641c in two different bands at $3.4 \mu \mathrm{m}$ (top), $4.6 \mu \mathrm{m}$ (bottom). In each image the yellow ellipse corresponds to the Fermi positional uncertainty region at $68 \%$ level of confidence. The white radio contours drawn from the NVSS image are overlaid on the WISE image at $3.4 \mu \mathrm{m}$. This is an example of a candidate association with a SFR that includes the open star cluster 165 from the Northern and Equatorial Milky Way catalog built with the Two Micron All-Sky Survey (2MASS) observations (Bica et al. 2003).

(Lane et al. 2014), both the Westerbork Northern Sky Survey (WENSS; Rengelink et al. 1997, - W) and the Westerbork in the Southern Hemisphere Source Catalog (WISH; De Breuck et al. 2002, - W), the Sydney University Molonglo Sky Survey (SUMSS; Mauch et al. 2003, - S), the ParkesMIT-NRAO Surveys (PMN; Wright et al. 1994, - Pm), the Texas Survey of Radio Sources at $365 \mathrm{MHz}$ (TEXAS; Douglas et al. 1996, - T) the Parkes Southern Radio Source catalog (PKS; Wright et al. 1990, - Pk), and the Lowfrequency Radio Catalog of Flat-spectrum Sources (LORCAT; Massaro et al. 2014a, - L).

For the radio counterparts (i.e., above $\sim 1 \mathrm{GHz}$ ), we searched the NRAO VLA Sky Survey (NVSS; Condon et al. 1998, - N), the VLA Faint Images of the Radio Sky at Twenty-Centimeters (FIRST; Becker et al. 1995; White et al. 1997, - F), the 87 Green Bank catalog of radio sources (87GB; Gregory \& Condon 1991, - 87) and 

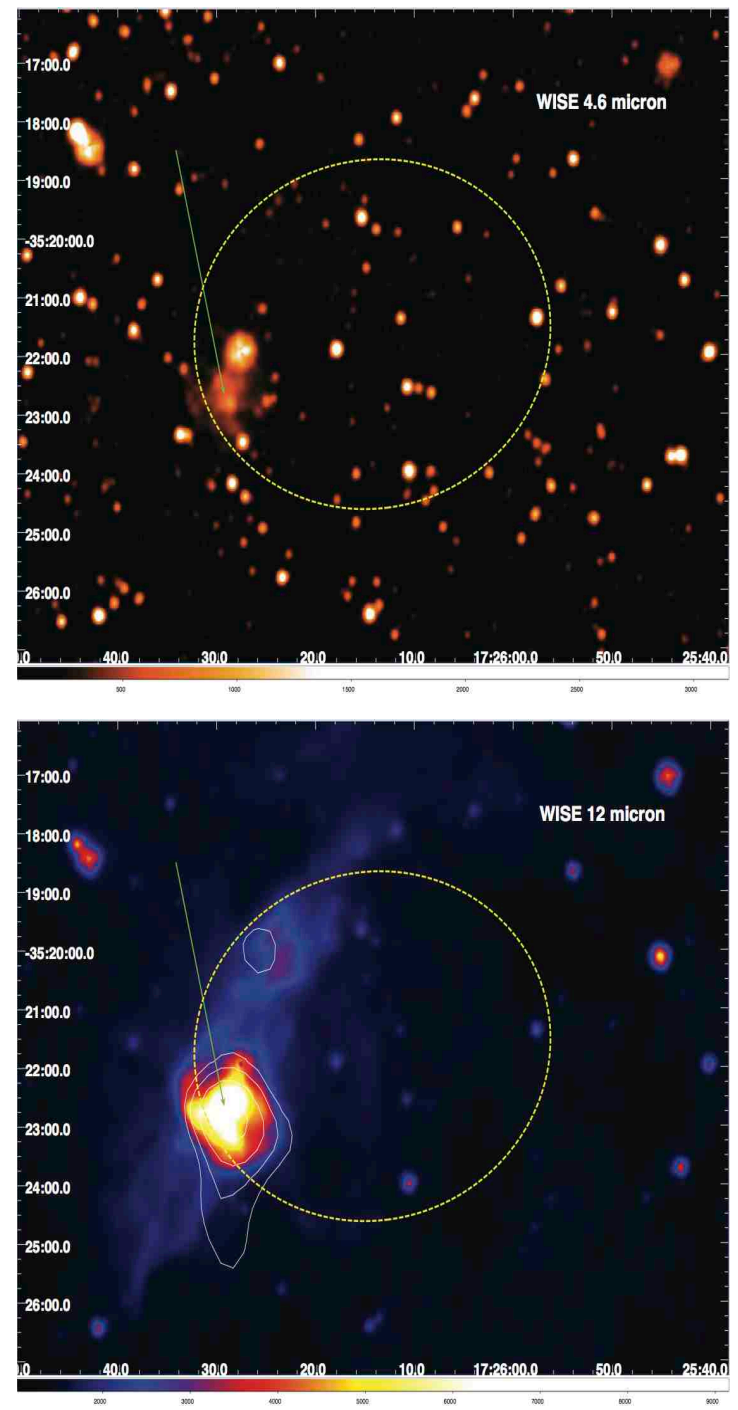

FIG. 3.- The WISE images of the Fermi source 1FGLJ1726.2-3521c in two different bands at $4.6 \mu \mathrm{m}$ (top), $12 \mu \mathrm{m}$ (bottom). In each image the yellow ellipse corresponds to the Fermi positional uncertainty region at $68 \%$ level of confidence. The white contours are overlaid on the WISE image at $12 \mu \mathrm{m}$ are the $1.4 \mathrm{GHz}$ radio emission from the NVSS. The radio-infrared extended structure, marked by the green arrow, clearly indicates that a shock occurred in the interstellar medium within the Fermi positional uncertainty region. This could be responsible for the $\gamma$-ray emission.

the Green Bank 6-cm Radio Source Catalog (GB6; Gregory et al. 1996, - GB), the Australia Telescope 20 GHz Survey (AT20G; Murphy et al. 2010, - A), the Combined Radio All-Sky Targeted Eight-GHz Survey (CRATES; Healey et al. 2007, - c) and the Australian Long Baseline Array (LBA) Calibrator Survey of southern compact extragalactic radio sources ${ }^{20}$ (LCS1 Petrov et al. 2011, - lcs).

For the infrared, we queried the WISE all-sky survey in the AllWISE Source catalog ${ }^{21}$ (Wright et al. 2010, - w) and the Two Micron All Sky Survey (2MASS; Skrutskie et al. 2006, - M) since each WISE source is automatically matched to the closest 2MASS potential counterpart (see Cutri et al. 2012, for details).

Then, we also searched for optical counterparts, with or

${ }^{20} \mathrm{http}: / /$ astrogeo.org/lcs/

21 http://wise2.ipac.caltech.edu/docs/release/allwise/
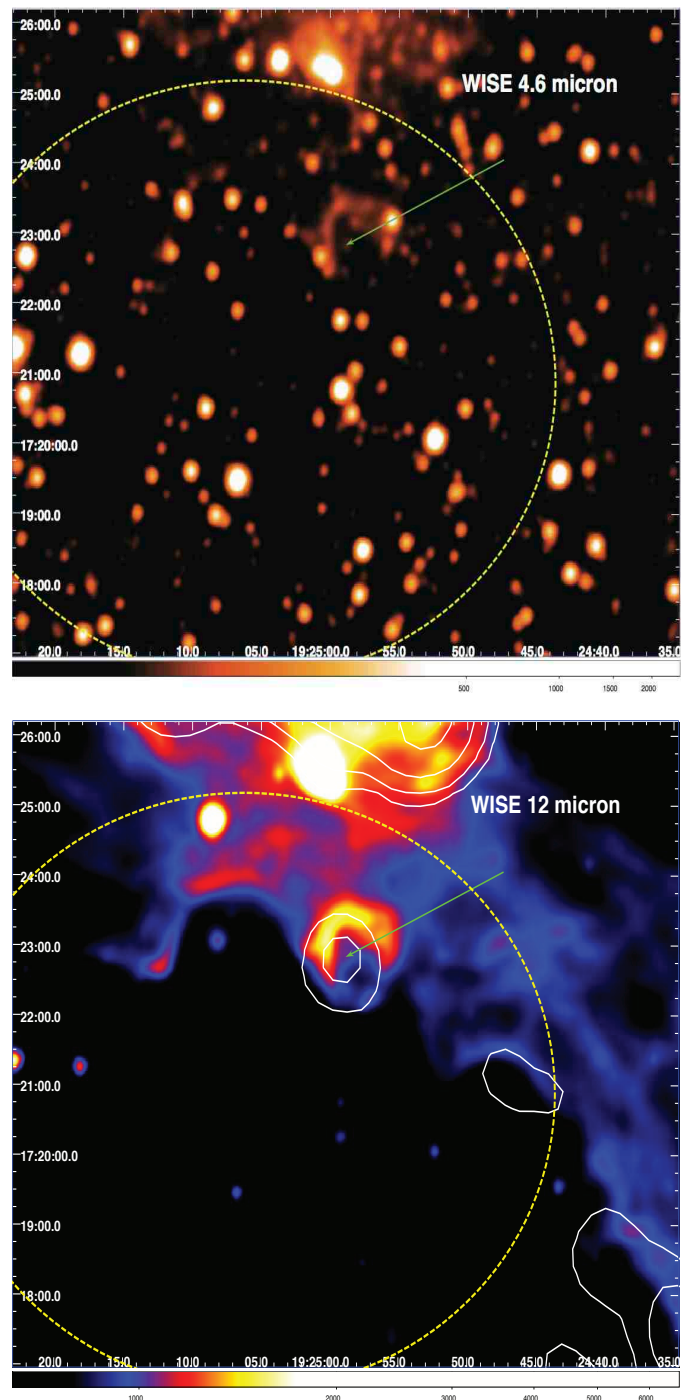

FIG. 4.- The WISE images of the Fermi source 1FGLJ1925.0+1720c in two different bands at $4.6 \mu \mathrm{m}$ (top), $12 \mu \mathrm{m}$ (bottom). In each image the yellow ellipse corresponds to the Fermi positional uncertainty region at $68 \%$ level of confidence. The white contours are overlaid on the WISE image at $12 \mu \mathrm{m}$ are the $1.4 \mathrm{GHz}$ radio emission from the NVSS. The ring-shaped structure, indicated by the green arrow, is extended for about $87^{\prime \prime}$ and resembles an unknown SNR or a PWN. It clearly lies within the Fermi positional uncertainty region and is also emitting in the radio band. This is a clear example of a candidate association with a SFR including a potential SNR/PWN for which additional multifrequency observations are necessary to confirm its origin.

without spectra available, in the Sloan Digitized Sky Survey Data Release 9 (SDSS DR9; e.g. Ahn et al. 2012, s), in the Six-degree-Field Galaxy Redshift Survey (6dFGS; Jones et al. 2004; Jones et al. 2009, - 6). We also queried the United States Naval Observatory (USNO)-B1 Catalog (Monet et al. 2003, - U) for the optical counterparts of the bcn and the unc sources and reported their magnitudes since they could be useful to plan future follow up observations.

In X-rays, we searched the ROSAT all-sky survey in both the ROSAT Bright Source Catalog (RBSC; Voges et al. 1999, - X) and the ROSAT Faint Source Catalog (RFSC; Voges et al. 2000, - X). as well as XMM-Newton Slew Survey (XMMSL; Saxton et al. 2008; Warwick et al. 2012, - X), the Deep Swift X-Ray Telescope Point Source Catalog (1XSPS; Evans et al. 2014, - x) the Chandra Source Catalog (CSC; Evans et al. 2010, - x) and Swift X-ray survey for all the Fermi 
TABLE 2

SOURCE CLASSIFICATION FOR THE $\gamma$-RAY COUNTERPARTS.

\begin{tabular}{|lc|}
\hline Class & code \\
\hline GALACTIC SOURCES & \\
\hline High mass X-ray binary & hmb \\
Globural cluster & glc \\
Nova & nov \\
Millisecond pulsar (MSP) & msp \\
Pulsar (PSR ) & psr \\
Pulsar wind nebula (PWN) & pwn \\
Binary star & bin \\
Star forming-HII region (SFR) & sfr \\
(for potential associations with unknown SNRs and/or PWNe and/or PSRs) & \\
Supernova remnant (SNR) & snr \\
\hline EXTRAGALACTIC SOURCES & \\
\hline BL Lac object (BZB) & bzb \\
Quasar radio loud with flat radio spectrum (BZQ) & bzq \\
Blazar of uncertain type (BZU) & bzu \\
Normal galaxy & gal \\
Radio galaxy & rdg \\
Starburst galaxy & sbg \\
Seyfert galaxy & sey \\
\hline UNCERTAIN CLASSIFICATION & \\
\hline Blazar candidate & bcn \\
Unclassified sources & unc \\
Unidentified gamma-ray source & ugs \\
\hline
\end{tabular}

UGSs (Stroh et al. 2013). We used the same designator for the X-ray catalogs of XMM-Newton, Chandra and Swift. These X-ray observatories perform only pointed observations thus there is the chance that the pointed observations present in their archives and related to the field of the Fermi source was not requested as follow up of the $\gamma$-ray source but for a different reason. Consequently, the discovery of the $X$-ray counterpart for an associated and/or identified source could be serendipitous.

To perform the searches over all the surveys and the catalogs cited above we considered the $1 \sigma$ positional uncertainties reported therein, with the only exceptions being the WISE all-sky survey and the SDSS DR9. In these two cases we searched the closest IR and optical counterpart within a maximum angular separation of $3^{\prime \prime} .3$ and of $1^{\prime \prime} .8$ for the AllWISE survey and the SDSS DR9, respectively. These values have been derived on the basis of the statistical analysis described in D'Abrusco et al. (2013) and Massaro et al. (2014b) developed following the approach also presented in Maselli et al. (2010) and Stephen et al. (2010).

To verify the presence of SFRs consistent with the positional uncertainty of the Fermi sources, we searched in the following surveys and catalogs: 1) the Sharpless catalog of HII regions (Sharpless 1959), 2) the HiI regions in the (InfraRed Astronomical Satellite (IRAS) point source catalog (Hughes \& MacLeod; Codella et al. 1994), 3) the radio survey of $\mathrm{H}_{\mathrm{II}}$ regions at $4.85 \mathrm{GHz}$ (Kuchar \& Clark 1997), 4) the new catalog of $\mathrm{H}_{\text {II }}$ regions in the Milky Way (Giveon et al. 2005), 5) the radio catalog of Galactic $\mathrm{H}$ II regions from decimeter to millimeter wavelength (Paladini et al. 2003) 6) the SCUBA imaging survey of ultra compact $\mathrm{H}_{\text {II }}$ regions (Thompson et al. 2006); 7) the recent WISE Catalog of Galactic $\mathrm{H}_{\text {II }}$ Regions (Anderson et al. 2014) 8) the QUaD Galactic Plane Survey (Culverhouse et al. 2011) 9) the $5 \mathrm{GHz}$ VLA survey of the Galacitc plane (Becker et al. 1994) and 10) the WSRTGP (Taylor et al. 1996). We remark that for the cross-matching performed between the SFR catalogs and the Fermi sources
TABLE 3

MULTIFREQUENCY NOTES FOR PULSARS.

\begin{tabular}{|cl|}
\hline Symbol & Comment \\
\hline $\mathrm{b}$ & PSR in a binary system \\
$\mathrm{e}$ & PSR detected in $\gamma$ rays by the Compton Gamma-ray Observatory \\
$\mathrm{g}$ & PSR discovered in LAT $\gamma$-ray data \\
$\mathrm{m}$ & MSP \\
$\mathrm{p}$ & Pulsar discovered by the Pulsar Search Consortium (PSC) \\
$\mathrm{r}$ & PSR discovered in the radio \\
$\mathrm{u}$ & PSR discovered using a Fermi-LAT seed position \\
$\mathrm{x}$ & PSR discovered in X-rays \\
\hline
\end{tabular}

we took into account the size of each $\mathrm{H}$ II region in combination with the Fermi uncertainty position.

To check for the presence of PSRs we used the Australia Telescope National Facility (ATNF) Pulsar Catalog (Manchester et al. 2005), the Catalogue of Galactic Supernova Remnants (Green 2009), and the census of high-energy observations of Galactic SNRs ${ }^{22}$ (Ferrand \& Safi-Harb 2012).

Table 4 summarizes all the notes derived from the multifrequency analysis. In particular, for the PSRs, we report the information shown in the Public List of LAT-Detected Gamma-Ray Pulsars ${ }^{23}$ and by the Pulsar Search Consortium (PSC) as well as from literature (e.g. Hobbs et al. 2004; $\quad$ Espinoza et al. 2013; Ray et al. 2012; Ray et al. 2013; Pletsch et al. 2012a; Pletsch et al. 2012b; Pletsch et al. 2013; Hanabata et al. 2014). Symbols used for their multifrequency notes are labelled as reported in Table 3 (see Abdo et al. 2013, for more details).

\section{THE REFINED ASSOCIATIONS FOR THE Fermi CATALOGS}

The refined associations for the 1FGL and the 2FGL catalog (hereinafter 1FGLR and 2FGLR, respectively) is presented in Table 4. For each source we report: the 1FGL name together with the 2FGL and the 1FHL ones as derived from the associations published in those catalogs, the counterpart name, and in 37 cases also an alternative association; the category of $\gamma$ ray source association; the class of each counterpart as found in the literature; notes derived from the multifrequency investigation; we also report whether the counterpart lies inside a SFR, and specifically for the PSRs in a PWN or a SNR. In addition, in Table 4 we also report if a PWN or a SNR contains a known PSR. The coordinates of each counterpart are also included in Table 4 together with the optical magnitudes in B and R band from the USNO-B1 Catalog (Monet et al. 2003). Within the multifrequency notes in Table 4 we also indicate if the spectral energy distribution (SED) of the source is shown in Takeuchi et al. (2013), if a redshift estimate is present in literature (indicating the reference) and if the radio counterpart has a flat radio spectrum (i.e., designated $\mathrm{rf}$ in the notes of Table 4 whenever $\alpha<0.5$ in the radio band). In the on-line version of the table we also report the infrared analysis flag (Wright et al. 2010) for those sources, classified as blazar-like with a counterpart in the AllWISE catalog within $3^{\prime \prime} .3$ as well as the column with the confirmed redshifts.

For the counterpart names we used the following priorities: if the source is a known blazar we adopted the Roma-BZCAT

\footnotetext{
${ }^{22}$ http://www.physics.umanitoba.ca/snr/SNRcat/

23 https://confluence.slac.stanford.edu/display/GLAMCOG/Public+List+of+LAT-Detecte
} 
nomenclature while for the known pulsars that of the ATNF Pulsar Catalog ${ }^{24}$ (Manchester et al. 2005). Radio galaxies, Seyfert galaxies and starburst names were indicated from the Third Cambridge Catalogue of Radio Sources and revised versions (3C and 3CR Edge et al. 1959; Bennett 1962; Spinrad et al. 1985), the New General catalog and the Messier catalog or using their radio names reported in one of the major surveys (see Section 5). Then Galactic sources as SNRs and PWNe have their names in Galactic coordinates as reported in the Catalogue of Galactic Supernova Remnants ${ }^{25}$ (Green 2009), while the most common names were used for globular clusters and binaries. The counterparts that were associated with SFRs were also labelled with their name in Galactic coordinates. Finally, the remaining uncertain sources were identified by their radio names whenever possible or by their ROSAT name one. A handful of counterparts were indicated using the nomenclature of optical catalogs. Adopting these choices in the names we remark that all the counterparts can be retrieved from the NASA/IPAC Extragalactic Database (NED) and SIMBAD archives without using their coordinates.

\subsection{The refined associations of the $1 F G L$}

The 1FGLR lists 111 identified sources, 880 associated, 306 candidate associations, and 154 UGSs. The sky distribution of the UGSs is shown in Figure 5 in comparison with the 1FGL associations in which there were 66 identified objects, 755 associated, and 630 unidentified. The results of our multifrequency analysis for the 1FGLR are summarized in Table 5 where we list all the sources identified, associated, and candidate associations for each class separately. We remark that the globular cluster $\mathrm{GCl} 94$ (alias M28) associated with 1FGL J1824.5-2449 contains a known MSP PSR J1824-2452A while 2MASS-GC01 lies in an SFR.

Among the PSRs and the MSPs are 21 sources that lie within a known PWN and 4 in SNRs as reported in the notes of Table 4. Twenty PSRs and MSPs out of 39 indicated as candidate associations in both Fermi catalogs have been identified after the release of the 2FGL catalog, as presented in the Public List of LAT-Detected Gamma-Ray Pulsars. This strongly supports our introduction of this new category of $\gamma$ ray sources. In five of the 10 associations of PWNe a PSR also has been found within the nebula and the same situation occurs for 8 SNRs.

We find that 113 1FGL sources have an SFR within their positional uncertainty regions obtained by combining in quadrature the $\gamma$-ray localization at $95 \%$ level of confidence with the size of the SFR. Three of these sources are the HMBs and one is the globular cluster 2MASS-GC01. Fifty-six of these 113 have been tentatively associated (i.e., indicated as candidate associations) with previously UGSs while the remaining Fermi objects all have PSRs, PWNe and/or a SNRs embedded in gas clouds and/or interacting with the interstellar medium $^{26}$. Among the 54 candidate associations with SFRs, two contain SNRs, one a PWN, and one a known PSR. However, the potential associations with SFRs could be used to refine the $\gamma$-ray Galactic diffuse emission models and the associations in future releases of the Fermi catalogs, as well as lead to the discovery of new PWNe and/or unknown SNRs.

\footnotetext{
${ }^{24}$ http://www.atnf.csiro.au/research/pulsar/psrcat/

25 http://www.mrao.cam.ac.uk/surveys/snrs/

26 see also http://astronomy.nju.edu.cn/ ygchen/others/bjiang/interSNR6.htm
}

None of the MSPs has been found lying within a known SFR while this occurs for 21 PSRs, 7 PWNe, and 18 SNRs.

It is worth noting that 1FGL J0503.2+4526, associated according to the 2FGL and the 2LAC analyses with an unclassified source, has as an alternative potential counterpart a SNR that includes a PSR. A similar situation occurs for 1FGL J1837.5-0659c and 1FGL J1846.8-0233c whose $\gamma$-ray emission could be ascribed to a PSR in a PWN. In addition, 1FGL J0622.2+3751 has as an alternative association with a PSR.

Within the unclassified candidate associations (i.e., unc), we noticed that 8 1FGLR sources are positionally consistent with SFRs. In addition, we also listed in the 1FGLR the tentative association of 1FGL J1653.6-0158 (also known as 2FGL J1653.6-0159) with the binary system that includes PSR J1653-0158 (Romani et al. 2014).

Finally, we remark that in the extragalactic sky the Small Magellanic Cloud (alias NGC 292) is identified with the Fermi source 1FGL J0101.3-7257 and classified as a normal galaxy while the Large Magellanic Cloud is associated with all the remaining five Fermi sources listed as normal galaxies.

\subsection{The refined associations of the $2 F G L$}

In the analysis of the 1FGLR we have already considered the associations for 1099 Fermi sources that are in common between the 1FGL and the 2FGL, however, we report the results for the whole refined $2 \mathrm{FGL}$ associations (hereinafter 2FGLR) in Table 5. As for the 1FGLR, the 2FGLR cata$\log$ is dominated by blazar-like sources in the extragalactic sky and by pulsars around the Galactic plane. The 2FGLR lists 126 identified sources as in the original catalog, 1176 associated, 282 candidate associations and 289 UGSs. The 2FGLR sources are classified as: 4 high mass X-ray binaries, 11 globular clusters, 51 MSPs, and 98 PSRs, 21 that lie in a PWN and 6 in an SNR, and 17 associated with a SFR indicated in our Table 4. In the case of 2FGLR, among the PSR candidate associations that are not in the 1FGLR only one source has been recently identified according to the Public List of LAT-Detected Gamma-Ray Pulsars (see also Abdo et al. 2013). There are also 9 PWNe and 66 sources associated with SNRs, 11 of them with a known PSR lying inside the remnant. Then we list 63 Fermi sources as candidate associations with SFRs, one also including a SNR. With respect to the 1FGL there is also a new association with a nova (e.g., Cheung et al. 2014).

The extragalactic sky includes 277 BZBs, 338 BZQs, 54 BZUs, 3 Seyfert galaxies, and 3 starburst galaxies. Four Fermi sources are associated with the $\gamma$-ray emission arising from the Large Magellanic cloud, as occurred for the objects classified as galaxy in the 1FGLR, and one with M31 (i.e., the Andromeda Galaxy) (Abdo et al. 2010d). In addition we find 271 blazar candidates (bcn), 320 Fermi unclassified sources and 289 UGSs. In Figure 6 we report the comparison between the sky distributions of all the UGSs previously listed in the $2 \mathrm{FGL}$ and the remaining ones determined in this refined association analysis.

\section{INFRARED COLORS OF $\gamma$-RAY BLAZAR CANDIDATES AND} UNCLASSIFIED SOURCES

As already done for the AGUs (Massaro et al. 2012a) and the UGSs (e.g., Paggi et al. 2013) listed in the 2FGL we performed a non-parametric analysis of the infrared colors for the Fermi sources classified as bcn and unc in the merged Fermi catalog 1FGLR+2FGLR. We used kernel density estimation 
TABLE 4

Refined associations of THE Fermi catalogs (First 30 Lines).

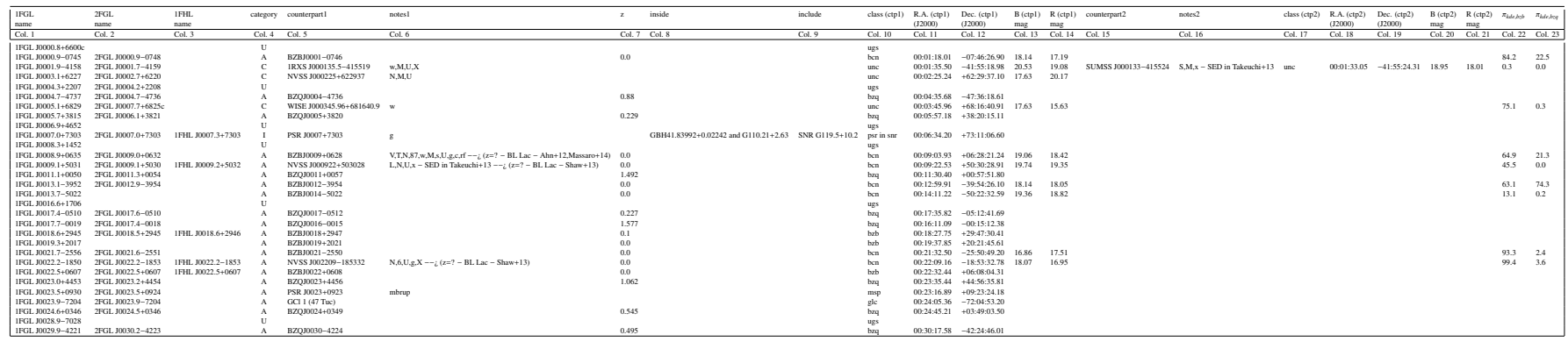

Column description. (1): 1FGL name; (2): 2FGL name; (3): 1FHL name; (4): $\gamma$-ray source category (I=identified; $\mathrm{A}=$ associated; $\mathrm{C}=$ candidate; $\mathrm{U}=$ unidentified; see Section 2 for details); (5): name of the first low-energy counterpart; (6): multifrequency notes on the first low-energy counterpart; (7): redshift estimate if the source is extragalactic (in the on line version of the catalog there is also a note if the $z$ measurement is uncertain); (8): notes if the first low-energy counterpart lies inside a SFR/SNR/PWN; (9): notes if the first low-energy counterpart includes a PSR/MSP; (10): source class for the first low-energy counterpart (see Section 4 for details); (11): R.A. (J2000) for the first low-energy counterpart; (12): Dec. (J2000) for the first low-energy counterpart; (13): B magnitude for the first low-energy counterpart taken from the USNO-B1 Catalog (Monet et al. 2003), only for the bcn and the unc sources; (14): R magnitude for the first low-energy counterpart taken from the USNO-B1 Catalog (Monet et al. 2003), only for the ben and the unc sources; (15): name of the second low-energy counterpart; (16): multifrequency notes on the second low-energy counterpart; (17): source class for the second low-energy counterpart (see Section 4 for details); (18): R.A. (J2000) for the second low-energy counterpart; (19): Dec. (J2000) for the second low-energy counterpart; (20): B magnitude for the second low-energy counterpart taken from the USNO-B1 Catalog (Monet et al. 2003), only for the ben and the unc sources; (21): R magnitude for the second low-energy counterpart taken from the USNO-B1 Catalog (Monet et al. 2003), only for the ben and the unc sources; (22): probability that the first low-energy counterpart has infrared colors similar to the known 1FGL BZBs estimated with the KDE method (see Section 7 for details); (23): probability that the first low-energy counterpart has infrared colors similar to the known 1FGL BZQs estimated with the KDE method (see Section 7 for details).

Symbols used for the multifrequency notes are all reported in Section 5 together with the references of the catalogs/surveys.

References for the optical spectra reported in the table: Ackermann et al. (2011), Ahn et al. (2012), Baker et al. (1995), Bauer et al. (2000), Bade et al. (1995), Beuermann et al. (1999), Bikmaev et al. (2008), Britzen et al. (2007), Drinkwater et al. (1997), Healey et al. (2008), Hewett \& Wild (2010), Hewitt \& Burbidge (1989), Johnston et al. (1995), Jones et al. (2004), Jones et al. (2009), Landoni et al. (2014) Landt et al. (2001), Lister et al. (2011), Mao (2011), Maza et al. (1995), Marti et al. (2004), Masetti et al. (2013), Massaro et al. (2014c) Mitton et al. (1977), Paggi et al. (2013), Quintana \& Ramirez (1995), Schwope et al. (2000), Shaw et al. (2013a), Shaw et al. (2013b), Stern \& Assef (2013), Stickel \& Kuehr (1996a,b) Titov et al. (2011), Vandenbroucke et al. (2010), Vettolani et al. (1989), Takeuchi et al. (2013), Thompson et al. (1990), Tsarevsky ert al. (2005), White et al. (1988).

1FGL unidentified gamma-ray sources

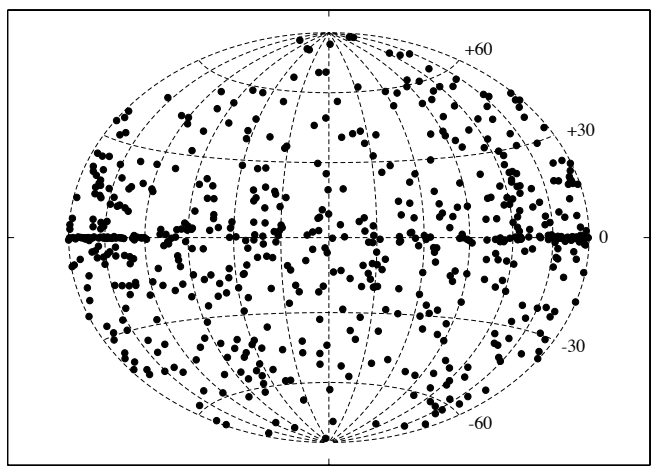

1FGLR unidentified gamma-ray sources

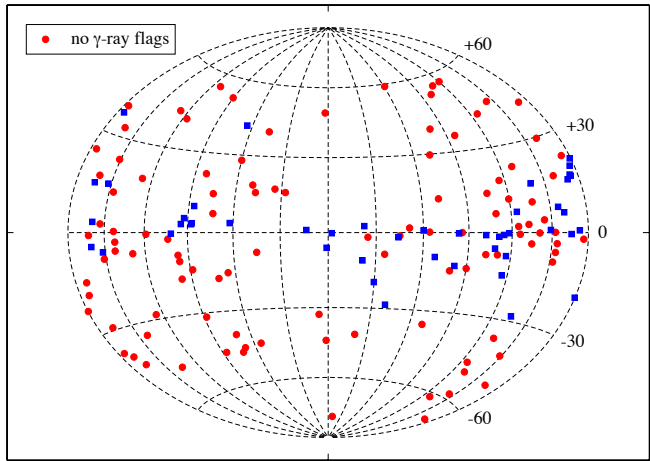

FIG. 5.- The all-sky distribution of the UGSs in the 1FGL (left) in comparison with those listed in the 1FGLR (right panel). Red circles mark those UGSs with no $\gamma$-ray analysis flags and blue squares those with a flag. The projection is Hammer-Aitoff projection in Galactic coordinates

(KDE; see e.g., D’Abrusco et al. 2009; Laurino et al. 2011, and reference therein) to verify the consistency of their infrared colors with the so-called WISE Gamma-ray Strip (e.g., Massaro et al. 2011a; Massaro et al. 2012a; Massaro et al. 2013c) The KDE technique allows us to estimate the probability function of a multivariate distribution and does not require any assumption about the shape of the "parent" distributions.

Consequently, for all the bcn and unc sources with a WISE counterpart we can provide an estimate of the probability $\pi_{k d e}$ that such source is consistent with the WISE Gammaray Strip. In particular we differentiated between $\pi_{k d e, b z b}$ and $\pi_{k d e, b z q}$ considering the comparison with the infrared colors of the BZB and BZQ subclasses, respectively. We considered as training samples to build the WISE Gammaray Strip(Massaro et al. 2011a; Massaro et al. 2013a) and to compare the infrared colors, the Roma-BZCAT blazars listed in both 1FGLR and 2FGLR. We remark that WISE counterparts of the bcn and of the unc sources with the infrared analysis flags $^{27}$ (Wright et al. 2010) have been considered in our $\begin{array}{lccccc}27 & \text { As for example contamination } & \text { and } & \text { con- } \\ \text { fusion from } & \text { nearby bright sources, see } & \text { e.g., } \\ \text { http://wise2.ipac.caltech.edu/docs/release/allsky/expsup/sec6_3a.html } & \end{array}$ 
2FGL unidentified gamma-ray sources

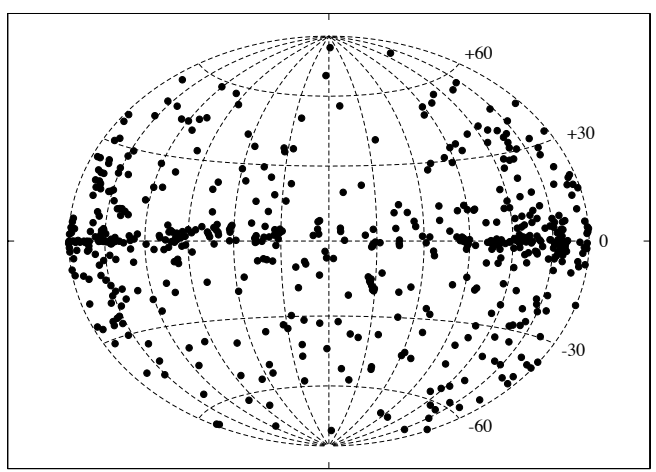

2FGLR unidentified gamma-ray sources

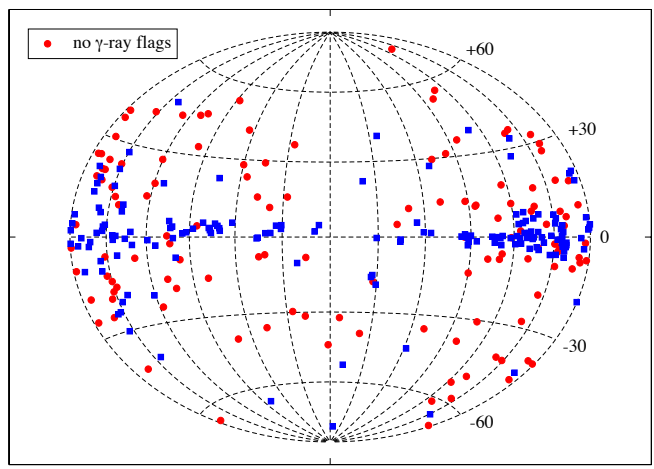

FIG. 6.- All-sky distribution of the UGSs in the 2FGL (left) in comparison with those listed in the 2FGLR (right panel). Red circles mark those UGSs with no $\gamma$-ray analysis flags and blue squares those with a flag.

TABLE 5

SUMMARY OF CATEGORIES FOR THE $\gamma$-RAY SOURCE ASSOCIATIONS IN THE REFINED ASSOCIATION LIST OF BOTH Fermi CATALOGS.

\begin{tabular}{|c|c|c|c|c|c|c|c|c|}
\hline \multirow[b]{2}{*}{ Class } & \multicolumn{3}{|c|}{ 1FGLR } & \multirow[b]{2}{*}{ Tot. } & \multicolumn{3}{|c|}{ 2FGLR } & \multirow[b]{2}{*}{ Tot. } \\
\hline & ID. & ASS. & CAN. & & ID. & ASS. & CAN. & \\
\hline GALACTIC & & & & & & & & \\
\hline bin & 0 & 1 & 0 & 1 & 0 & 1 & 0 & 1 \\
\hline $\mathrm{hmb}$ & 3 & 0 & 1 & 4 & 4 & 0 & 0 & 4 \\
\hline glc & 0 & 9 & 0 & 9 & 0 & 11 & 0 & 11 \\
\hline nov & 0 & 0 & 0 & 0 & 1 & 0 & 0 & 1 \\
\hline $\mathrm{msp}$ & 26 & 16 & 8 & 50 & 26 & 17 & 8 & 51 \\
\hline psr & 53 & 8 & 31 & 92 & 56 & 12 & 30 & 98 \\
\hline pwn & 0 & 8 & 2 & 10 & 2 & 6 & 1 & 9 \\
\hline snr & 3 & 38 & 5 & 46 & 6 & 55 & 5 & 66 \\
\hline sfr & 0 & 0 & 54 & 54 & 0 & 0 & 63 & 63 \\
\hline \multicolumn{9}{|c|}{ EXTRAGALACTIC | } \\
\hline bzb & 7 & 231 & 1 & 239 & 7 & 270 & 0 & 277 \\
\hline bzq & 15 & 271 & 1 & 287 & 18 & 320 & 0 & 338 \\
\hline bzu & 2 & 46 & 0 & 48 & 2 & 52 & 0 & 54 \\
\hline gal & 1 & 5 & 0 & 6 & 2 & 4 & 0 & 6 \\
\hline rdg & 0 & 5 & 0 & 5 & 1 & 7 & 0 & 8 \\
\hline sbg & 0 & 3 & 0 & 3 & 0 & 3 & 0 & 3 \\
\hline sey & 0 & 2 & 0 & 2 & 0 & 3 & 0 & 3 \\
\hline \multicolumn{9}{|l|}{ UNCERTAIN } \\
\hline bcn & 0 & 188 & 25 & 213 & 0 & 242 & 29 & 271 \\
\hline unc & 1 & 49 & 178 & 228 & 1 & 173 & 146 & 320 \\
\hline ugs & 0 & 0 & 0 & 154 & 0 & 0 & 0 & 289 \\
\hline
\end{tabular}

Col. (1) Source class. Col. (2) ID.= identified. Col. (3) ASS.= associated. Col. (4) CAN.= candidate associations.

analysis and their flags are reported in Table 4 together with the probabilities derived from the KDE analysis.

In Figure 7, the isodensity contours drawn from the KDE density probabilities are plotted for the Fermi-Roma-BZCAT blazars in the [3.4]-[4.6]-[12] $\mu \mathrm{m}$ color-color diagram. The IR colors of the sources listed in the refined association list of the Fermi catalogs classified as bcn and unc are also shown. Values of $\pi_{k d e, b z b}$ and $\pi_{k d e, b z q}$ greater than $5 \%$ indicate that the source has infrared colors consistent with the portion WISE Gamma-ray Strip constituted by the BZBs and by the BZQs, respectively, at $95 \%$ confidence level.

Our analysis confirms that 419 out of 499 total ben (262) and unc (237) sources in the refined and merged associations list of the Fermi catalogs are consistent with the WISE

for additional details
Gamma-ray Strip at $95 \%$ confidence level. For the unc sources this situation occurs for 172 sources out of $237 \mathrm{ob}$ jects while for the bcn in 242 out of 262 cases. We remark that 374 out of the 499 sources analyzed with the KDE are associated, 1 is identified, and the remaining 124 are candidate associations. This analysis supports the introduction of the category for the candidate associations, since a large fraction of the bcn and the unc with a WISE counterpart are likely to be blazar-like sources being consistent with the WISE Gammaray Strip.

In our analysis, we took into account the correction for Galactic extinction for all the WISE magnitudes according to the Draine (2003) relation. As shown in D'Abrusco et al. (2013), this correction affects only marginally the [3.4]-[4.6] $\mu \mathrm{m}$ color, mostly at low Galactic latitudes (i.e., $|b|<15^{\circ}$ ).

\section{OPTICAL SPECTROSCOPIC OBSERVATIONS OF $\gamma$-RAY BLAZAR CANDIDATES}

The total number of Fermi sources listed uniquely in the 1FGLR and in the 2FGLR are 2219. We found spectroscopic information for 177 of them not reported in the previous 1FGL and 2FGL catalogs, including 8 observed during our spectroscopic campaign (see below).

We have been able to confirm spectroscopically $117 \mathrm{BL}$ Lacs, 21 with firm redshift estimates and 27 quasars (QSOs); an additional eight sources appear to have a BL Lac nature but the lack of good optical spectra in the literature or, if a spectrum is available, a low signal to noise ratio did not allow us to verify their natures. Thus 24 out of these 177 sources still have an uncertain classification and an uncertain redshift estimate. In particular among them we found 11 sources listed as blazars in Ackermann et al. (2011) plus one listed in Healey et al. (2007) with no optical spectra published or described in literature. These 177 sources are all listed in Table 4 as bcn' with the only exception being one radio source that appears to be a radio galaxy; in the table notes we report their possible classifications and their redshift estimates with a question mark (?) indicating that further investigation is required.

Optical spectroscopic observations of four candidate associations and of three associated with unclassified sources (unc) were performed with the 2.1-m telescope of the Observatorio Astronómico Nacional (OAN) in San Pedro Mártir (México) on the nights between 28 June and 2 July 2014 

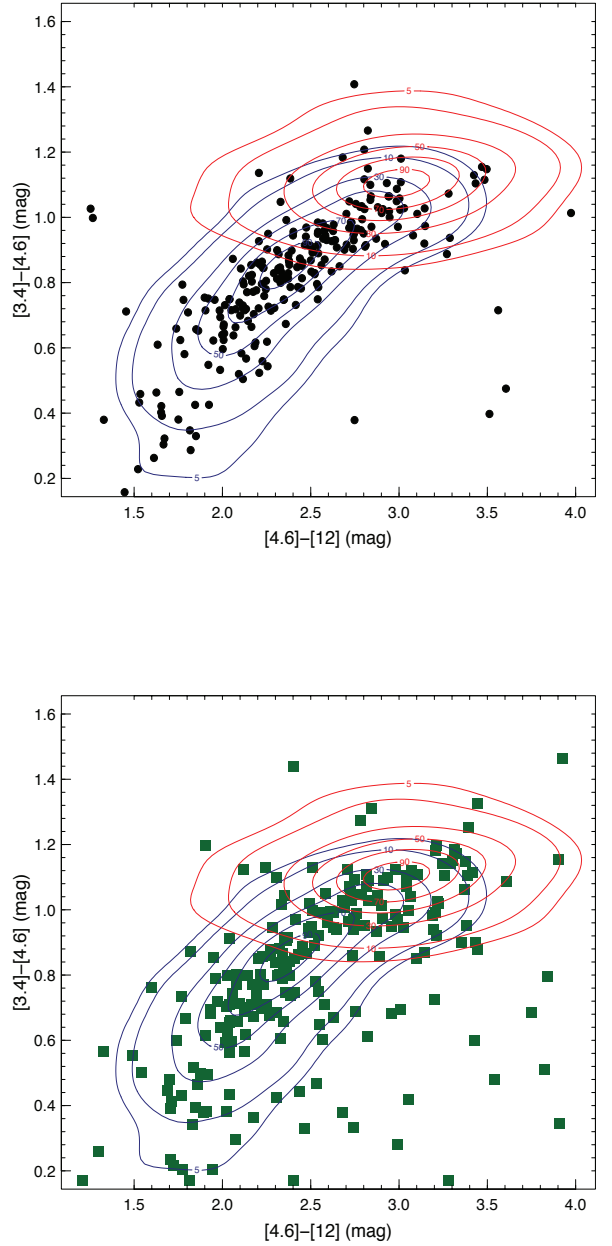

FIG. 7.- The isodensity contours drawn from the KDE technique for the Fermi-Roma-BZCAT blazars (i.e., training sample) that constitute the WISE Gamma-ray Strip (Massaro et al. 2011a) in the [3.4]-[4.6]-[12] $\mu \mathrm{m}$ colorcolor plot. Blue contours were computed from the infrared colors of the BZBs while the red ones were derived from the BZQs. The infrared colors of the sources classified as ben (top - black circles) and unc (bottom - green squares) are also reported to show their consistency with the WISE Gammaray Strip. The numbers appearing close to each contour correspond to the values of $\pi_{k d e}$ in both panels. The last contour correspond to $95 \%$ confidence level (see Section 7 for details). A similar analysis for the active galaxies of uncertain type listed in the 2FGL was already performed in Massaro et al. (2012a). Here this study has been repeated for both the 1FGLR and 2FGLR lists. refined association list of the Fermi catalogs.

(UT) as reported in Table 6. The telescope carries a Boller \& Chivens spectrograph and a $1024 \times 1024$ pixel E2V-4240 CCD. The spectrograph was tuned in the $4000 \div 8000 \AA$ range (grating $3001 / \mathrm{mm}$ ), with a resolution of $4.5 \AA$ per pixel, which corresponds to $8 \AA$ (Full-width-half-maximum), and a 2'.5 slit. Data were wavelength calibrated using Copper-HeliumNeon-Argon lamps, while for flux calibration spectrophotometric standard stars were observed twice during every night of the observing run.

In addition we also observed the $\gamma$-ray blazar candidate BZB J2340+8015 associated with 1FGL J2341.6+8015 at the Observatorio Astrofisico Guillermo Haro (OAGH) located at Cananea, Sonora in México. The telescope was equipped with a spectrograph Boller \& Chivens with a CCD SITe $1 \mathrm{k} \times 1 \mathrm{k}$, tuned in the $4000 \div 7000 \AA$ range (grating $150 \mathrm{l} / \mathrm{mm})$. We used a slit width of $2^{\prime \prime} .5$ with a resolution $\sim 15 \AA$ (FWHM).

The data reduction for both telescopes and instruments was carried out using the Image Reduction and Analysis Facility (IRAF) package developed by the National Optical Astronomy Observatory, including bias subtraction, spectroscopic flat fielding, optimal extraction of the spectra and interpolation of the wavelength solution. All spectra were reduced and calibrated employing standard techniques in IRAF and our own IDL routines (see also Matheson et al. 2008, for additional details).

These eight sources for which new spectroscopic data are provided in our analysis are listed in Table 4 as $\gamma$-ray blazar candidates (i.e., bcn). Five of them belong to the 1FGLR and are all classified as BL Lac objects according to our analysis; for three of them the presence of several spectral features allowed us to determine their redshifts. The log of the spectroscopic observations and the results of our analysis are reported in Table 6, while in Figure 8, Figure 9, Figure 10 and Figure 11 we show their optical spectra together with their finding charts. The remaining three sources are two quasars corresponding to 2FGL J1848.6+3241 and 2FGL J2021.5+0632 as shown in Figures 12 and 13 at redshifts 0.981 and 0.217 , respectively, and 2FGL J2031.0+1938, a BL Lac object with an uncertain redshift since there is a unique emission line visible, potentially identified as Mg II (see Figure 14).

During our observing nights we also obtained the spectra for two 2FGL sources, 2FGL J1719.3+1744 and 2FGL $\mathrm{J} 1801.7+4405$, each with uncertain redshift as reported in the Roma-BZCAT (see the figures in the Appendix). We were able to confirm the redshift for 2FGL J1801.7+4405 while 2FGL J1719.3+1744 (alias BZB J1719+1745) is completely featureless. Results for these two additional spectra are also reported in Table 6. We also observed 1FGL $\mathrm{J} 1942.7+1033$, which is a BL Lac object already classified in literature (Tsarevsky et al. 2005; Masetti et al. 2013, and Appendix for the figure), and 1FGL J2300.4+3138 for which a series of unidentified absorption features are clearly visible in its optical spectrum. Assuming that these unidentified absorption features are due to $\mathrm{Mg}_{\text {II }}$ intervening systems along the line of sight, the source should lie at redshift $\geq 0.96$. This source was in the sample observed by Shaw et al. (2013a) but we cannot confirm their results since we did not find any spectral feature identifiable as C IV (see Figure 15). Finally we also report the spectrum of BZB J2340+8015 associated with 1FGL J2341.6+8015 observed at OAGH that was originally classified as a BL Lac candidate in the Roma-BZCAT at redshift 0.274 . We have been able to confirm the BL Lac nature of this source but not its redshift due to its featureless spectrum (see Figure 16). All our finding charts are from the Digitized Sky Survey ${ }^{28}$.

\section{COMPARISON WITH STATISTICAL ANALYSES}

We compared our multifrequency analysis on the candidate associations with the results obtained via statistical analyses based on the Classification Tree (CT) and on the Logistic Regression (LR) procedures performed on the 1FGL sources (Ackermann et al. 2012) and using the Random Forest algorithm, named sybil, presented by Mirabal et al. (2012) ${ }^{29}$ for the 2FGL sources.

\footnotetext{
${ }^{28}$ http://archive.eso.org/dss/dss

29 http://www.gae.ucm.es/ mirabal/sibyl.html
} 


\section{FGL J1548.7+6311}
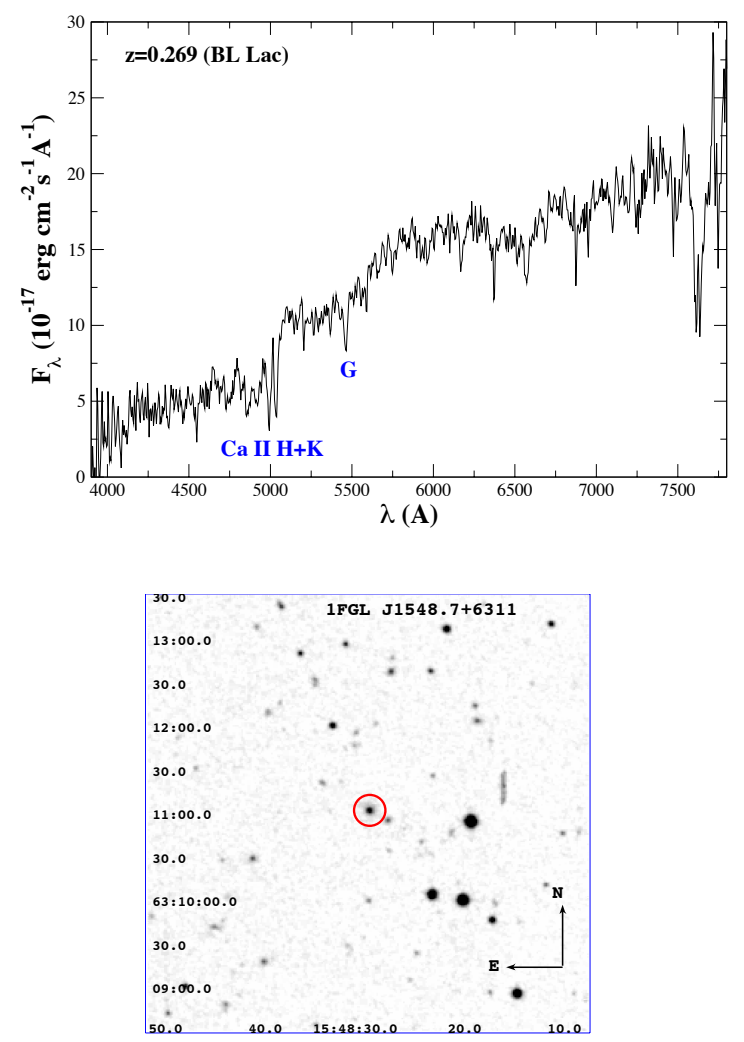

FIG. 8.- Upper panel) The optical spectra of the counterpart associated with 1FGL J1548.7+6311 observed at OAN in San Pedro Mártir (México) on 28 June 2014. The absorption features identified as $\mathrm{Ca}$ II $\mathrm{H} \& \mathrm{~K}$ and the $\mathrm{G}$ band used to determine its redshift are marked. The source has been classified as a BL Lac. (Lower panel) The $5^{\prime} \times 5^{\prime}$ finding chart from the Digitized Sky Survey (red filter). The potential counterpart of 1FGL J1548.7+6311, the target of our observation, is indicated by the red circle.

TABLE 6

LOG AND RESULTS OF OPTICAL SPECTROSCOPIC OBSERVATIONS.

\begin{tabular}{|c|c|c|c|c|c|}
\hline $\begin{array}{l}\text { Fermi } \\
\text { Name }\end{array}$ & $\begin{array}{l}\text { Obs. Date } \\
\text { yyyy-mm-dd }\end{array}$ & $\begin{array}{c}\text { Exposure } \\
(\mathrm{sec})\end{array}$ & Class & $z$ & $\begin{array}{l}\text { Spectral } \\
\text { Features }\end{array}$ \\
\hline 1FGL J1548.7+6311 & 2014-06-28 & $2 \times 1800$ & BL Lac & 0.269 & Ca п $\mathrm{H} \& \mathrm{~K}, \mathrm{G}, \mathrm{H} \beta, \mathrm{MgI}$ \\
\hline 1FGL J1844.1+1547 & 2014-06-29 & $2 \times 1800$ & BL Lac & ? & none \\
\hline 1FGL J2014.4+0647 & 2014-06-29 & $2 \times 1800$ & BL Lac & 0.341 & Cа п $\mathrm{H} \& \mathrm{~K}, \mathrm{G}, \mathrm{H} \beta, \mathrm{MgI}$ \\
\hline 1FGL J2133.4+2532 & 2014-06-29 & $2 \times 1800$ & BL Lac & 0.294 & Ca I $\mathrm{H} \& \mathrm{~K}, \mathrm{G}, \mathrm{H} \beta, \mathrm{MgI}$ \\
\hline 2FGL J1848.6+3241 & 2014-07-02 & $2 \times 1800$ & QSO & 0.981 & $\mathrm{Mg}$ II, [OII] $\lambda 3727$ \\
\hline 2 FGL J2021.5+0632 & 2014-07-02 & 1800 & QSO & 0.217 & $\mathrm{H} \gamma, \mathrm{H} \beta,[\mathrm{OIII}] \lambda 4959 \& \lambda 5007$ \\
\hline 2FGL J2031.0+1938 & 2014-07-01 & $2 \times 1800$ & BL Lac & $0.668 ?$ & $\mathrm{Mg}$ I? \\
\hline 2FGL J1719.3+1744 & 2014-06-30 & $2 \times 1800$ & BL Lac & ? & none \\
\hline 2FGL J1801.7+4405 & 2014-06-30 & $2 \times 1800$ & QSO & 0.663 & $\mathrm{Mg}$ II, [OII] $\lambda 3727$ \\
\hline 1FGL J1942.7+1033 & 2014-06-29 & $2 \times 1800$ & BL Lac & ? & none \\
\hline 1FGL J2300.4+3138 & 2014-07-01 & $2 \times 1800$ & BL Lac & ? & unid. abs. systems \\
\hline 1FGL J2341.6+8015 & 2014-08-28 & $3 \times 1800$ & BL Lac & ? & none \\
\hline
\end{tabular}

For the 1FGL sources the analysis executed with both the CT and the LR statistical methods assesses the probability of correct classification based on fitting a model form to the Fermi data. The result is a PSR-like or an AGN-like classification, occurring when the $\gamma$-ray source properties are likely more consistent with those of a pulsar or an active galaxy, respectively; a similar prediction is also made for the $2 \mathrm{FGL}$ sources analyzed by the sybil algorithm. Thus, all these meth-
1FGL J1844.1+1547
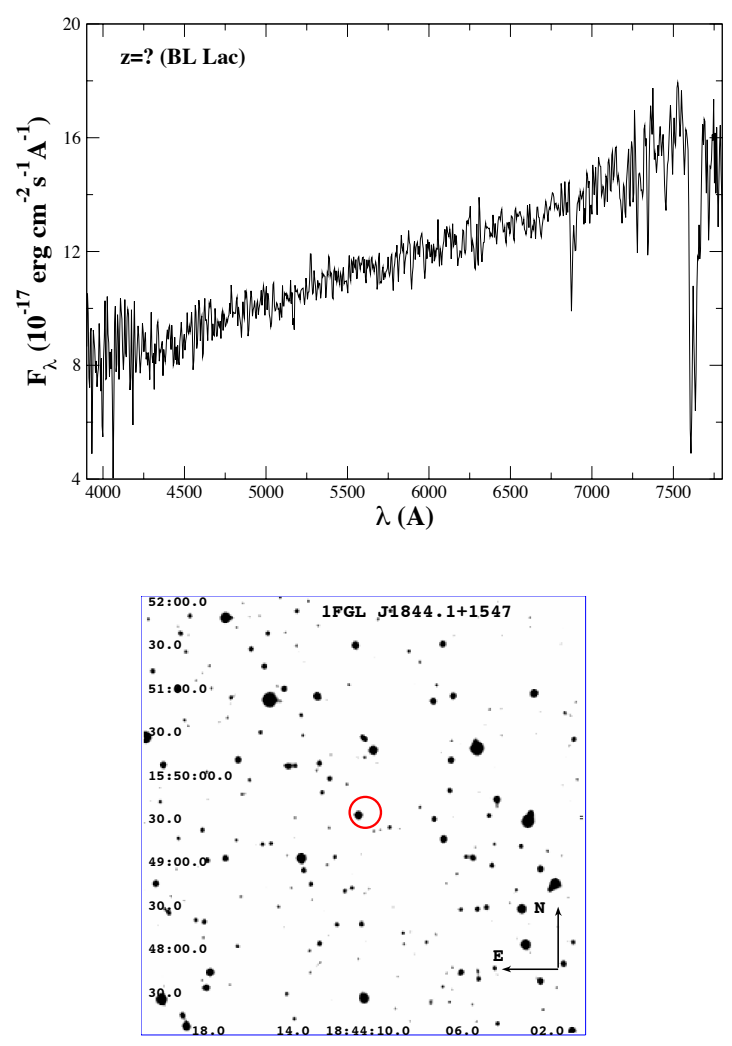

FIG. 9.- Upper panel) The optical spectra of the counterpart associated with 1FGL J1844.1+1547 observed at OAN in San Pedro Mártir (México) on 29 June 2014. The source has been classified as a BL Lac on the basis of its featureless continuum. (Lower panel) The $5^{\prime} \times 5^{\prime}$ finding chart from the Digitized Sky Survey (red filter). The potential counterpart of 1FGL $\mathrm{J} 1844.1+1547$, the target of our observation, is indicated by the red circle.

ods provide a classification of the potential counterpart on the basis of the $\gamma$-ray properties (e.g., spectral shape, variability, etc.) but cannot permit to locate it. Consequently we can only verify if a candidate association that appear to be a blazar or a pulsar have the corresponding $\gamma$-ray source classified as AGNlike or PSR-like by the statistical analyses cited above.

There are 286 candidate associations of type bzb in the 1FGLR that were analyzed in Ackermann et al. (2012). Within this sample we found that there is only one bzb and one hmb, the first statistically classified as AGN-like while the second as a PSR-like. Then there are 24 sources classified as bcn, with 23 of them indicated as AGN-like while the last is classified as PSR-like.

Among the Galactic sources, there are:

- 7 sources, classified by us as potential MSPs, four appear to be PSR-like while 3 show $\gamma$-ray properties more similar to AGNs (i.e., AGN-like).

- 25 out of 29 Galactic sources classified are in our analysis as PSRs are confirmed PSR-like by statistical methods while 4 are indicated as AGN-like;

- 2 PWN candidate associations, both resembling PSRlike sources in $\gamma$-rays.

- 5 sources reported in our Table 4 as SNRs, four are classified as PSR-like objects and one has an uncertain sta- 
1FGL J2014.4+0647
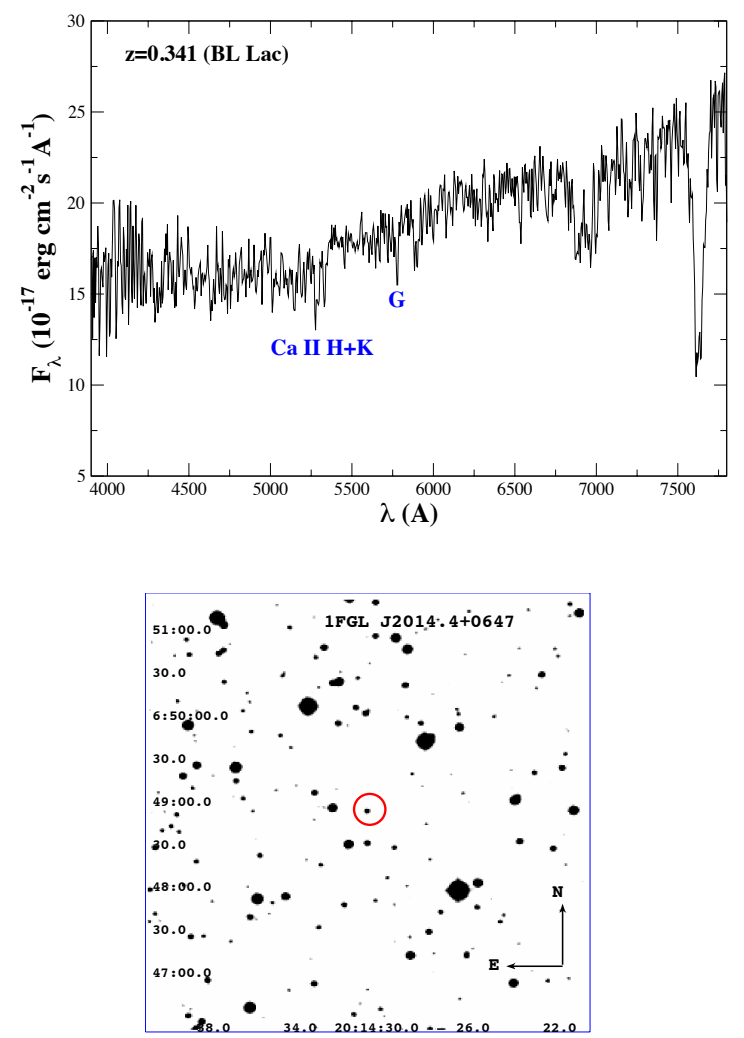

FIG. 10.- Upper panel) The optical spectra of the counterpart associated with 1FGL J2014.4+0647 observed at OAN in San Pedro Mártir (México). The source was observed twice on 29 June 2014 and on 30 June 2014 and both spectra are shown. The absorption features identified as $\mathrm{Ca}$ II $\mathrm{H} \& \mathrm{~K}$ and the $\mathrm{G}$ band used to determine its redshift are marked. The source has been classified as a BL Lac. Lower panel) The $5^{\prime} \times 5^{\prime}$ finding chart from the Digitized Sky Survey (red filter). The potential counterpart of 1FGL J2014.4+0647 is indicated by the red circle.

tistical classification (i.e., indicated as conflict in Ackermann et al. 2012).

- 33 out of 47 candidate associations classified as SFRs are PSR-like while only 9 appear to be classifiable as AGN-like by the statistical methods; the remaining five are unclassified.

The comparison between our candidate associations in the Galactic plane and the results of the statistical analyses supports the relevance of searching for the presence of SFRs within the Fermi positional uncertainty region where PSRs, SNRs, PWNe could be embedded therein or shocked regions could be potential sources of $\gamma$-rays not yet confirmed. Finally, in the sample of 170 unc sources, classified as candidate associations by our analysis, 125 of them appearing to be AGN-like while 40 are indeed PSR-like; the remaining five are conflicts between the LR and the CT methods. In particular, four out of eight unc sources having a SFR consistent with the Fermi position are classified as PSR-like on the basis of their $\gamma$-ray behavior.

In the case of the 2FGLR, we investigated 133 candidate associations with the sybil procedure, and classified them accordingly. Of these, in the sample of 107 Fermi sources that are classified as AGN-like by sybil: 24 are bcn, 78 are unc
1FGL J2133.4+2532
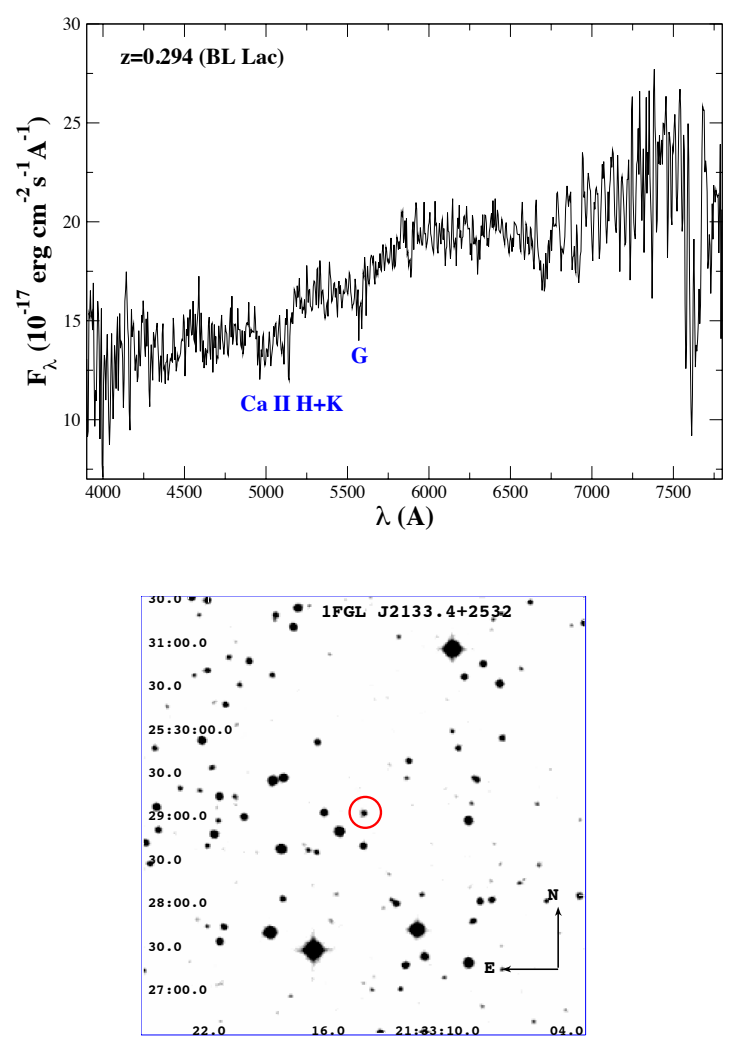

FIG. 11.- Upper panel) The optical spectra of the counterpart associated with 1FGL J2133.4+2532 observed at OAN in San Pedro Mártir (México) on 29 June 2014. The absorption features identified as $\mathrm{Ca}$ II $\mathrm{H} \& \mathrm{~K}$ and the $\mathrm{G}$ band used to determine its redshift are marked. The source has been classified as a BL Lac. Lower panel) The $5^{\prime} \times 5^{\prime}$ finding chart from the Digitized Sky Survey (red filter). The potential counterpart of 1FGL J2133.4+2532 is indicated by the red circle.

type; then the remaining objects are two SFRs, three PSRs, seven MSPs (three expected to be PSR-like $\gamma$-ray sources) and five SFRs all classified as AGN-like.

Finally, we highlight that the results of the statistical analyses are in agreement with the classification proposed in the refined association lists of both Fermi catalogs.

\section{GAMMA-RAY CONNECTIONS}

\subsection{The radio- $\gamma$-ray connection}

Many attempts were made in the past to test for correlations between the radio and $\gamma$-ray emissions of AGNs and in particular for blazars (e.g., $\quad$ Stecker et al. 1993; $\quad$ Padovani et al. 1993; Salamon \& Stecker 1994; Taylor et al. 2007). This connection was also used before the Fermi era to search for counterparts of the $\gamma$-ray sources (Mattox et al. 1997), so motivating all the past and present radio follow up campaigns for the Fermi sources (e.g., Petrov et al. 2013; Schinzel et al. 2014).

However, biases and selection effects have to be taken into account to prove this correlation properly, since it is important to address intrinsic source variability, biases due to redshift dependence (Elvis et al. 1978), the "common distance" bias (Pavlidou et al 2011), problems related to source misidentifications and incorrect associations, to name a few. All these issues can mimic a correlation (Mucke et al. 1997). 
2FGL J1848.6+3241
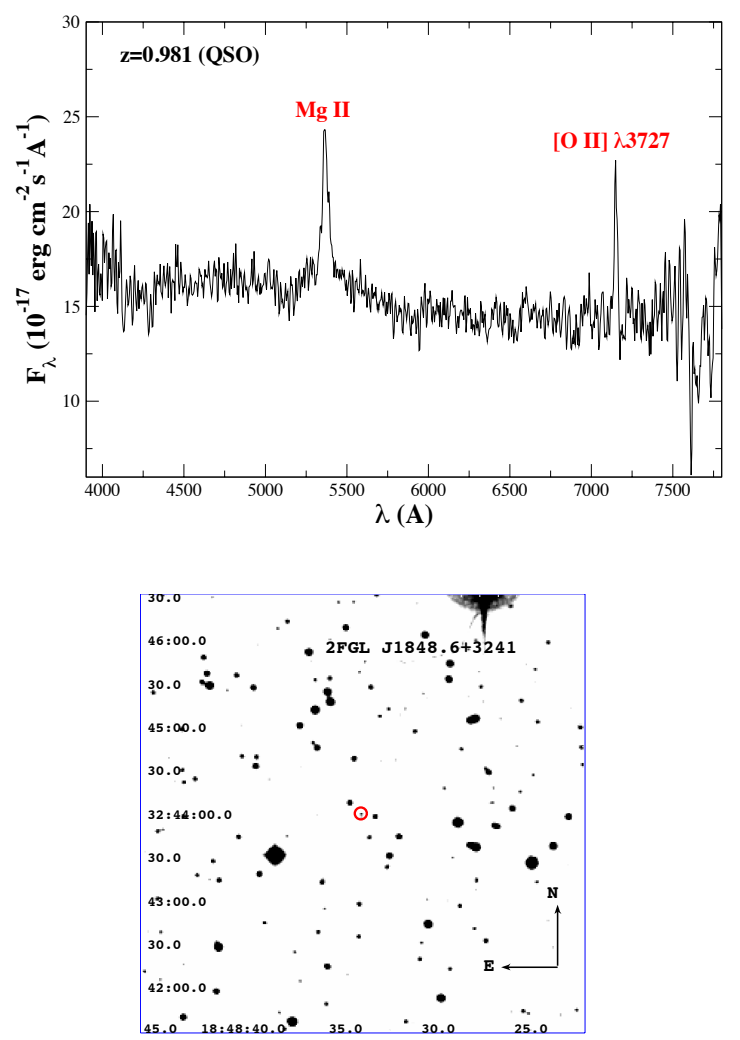

FIG. 12.- Upper panel) The optical spectra of the counterparts associated with 2FGL J1848.6+3241 observed at OAN in San Pedro Mártir (México) on 2 July 2014. The emission lines marked allowed us to estimate their redshift. The source has been classified as a QSO. Lower panel) The $5^{\prime} \times 5^{\prime}$ finding chart from the Digitized Sky Survey (red filter). The potential counterpart of 2FGL J1848.6+3241 is indicated by the red circle.

Since the launch of Fermi several investigations have also been performed to search for a definitive answer regarding the existence of the radio- $\gamma$-ray connection (Ghirlanda et al. 2010; Ghirlanda et al. 2011; Mahony et al. 2010) until it was proved and described accurately in Ackermann et al. (2011b). Recently we also suggested that a link between the radio and the $\gamma$-ray emissions in blazars can be extended well below $\sim 1$ GHz (Massaro et al. 2013b; Nori et al. 2014; Massaro et al. 2013d).

Given the new multifrequency analysis carried out here and the candidate associations, in particular for the bcn class we illustrate the current status of the radio- $\gamma$-ray connection. To this end we show both the flux-flux and the luminosityluminosity scatter plots for the blazars in our merged refined list of Fermi associations (see Figure 17 and Figure 18).

A study of the correlations is out of the scope of this paper. Our main goal is verify that sources classified as bcn are in agreement with the behavior of blazars in these parameter spaces. It is worth noting that we divided our sample of blazar-like sources in two subsamples: the northern one and the southern one. the former includes all the blazars (i.e., bzb, bzq, and bcn classes) that have a radio counterpart within the NVSS footprint while in the latter the subsample includes those in the area covered by the SUMSS. We distinguish these two samples since the radio surveys were carried out at differ-
2FGL J2021.5+0632
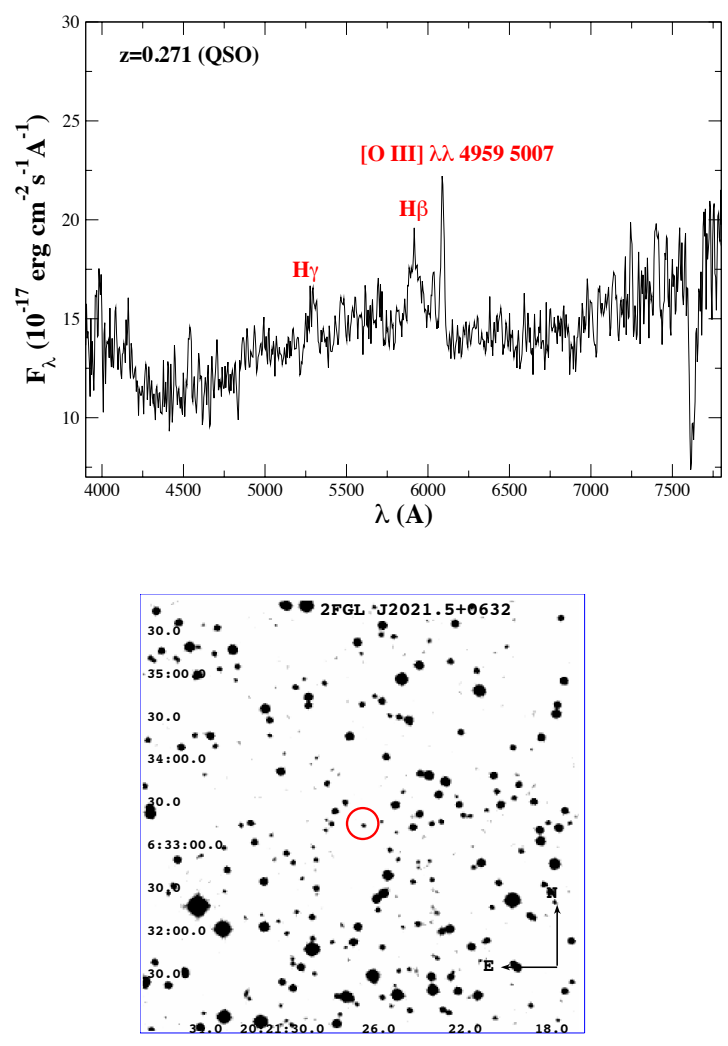

FIG. 13.- Upper panel) The optical spectra of the counterparts associated with 2FGL J2021.5+0632 observed at OAN in San Pedro Mártir (México) on 2 July 2014. The emission lines marked allowed us to estimate their redshift. The source has been classified as a QSO. Lower panel) The $5^{\prime} \times 5^{\prime}$ finding chart from the Digitized Sky Survey (red filter). The potential counterpart of 2FGL J2021.5+0632 is indicated by the red circle.

ent frequencies.

Finally, we remark that only blazars with a firm redshift estimate as reported in our merged list of refined associations are shown in Figure 18. According to this figure we could expect most of the ben sources to be BL Lac objects, although this has to be confirmed with optical spectroscopic observations.

\subsection{The infrared- $\gamma$-ray connection}

D'Abrusco et al. (2012) studied the Fermi blazar sample listed in the 2FGL and lying in the footprint of the WISE Preliminary survey and reported the discovery of a correlation between their infrared and their $\gamma$-ray spectral indices. This is directly related to their peculiar infrared colors and the WISE Gamma-ray Strip (Massaro et al. 2011a).

Here we report an updated scatterplot for the spectral indices (see Figure 19) as originally presented in D'Abrusco et al. (2012). The correlation the spectral shapes in the infrared and in $\gamma$-rays is still present and as occurred in the case of the radio - $\gamma$-ray scatter plot (see Section 10.1) the locations of the bcn sources appear to be more consistent with the BL Lac population. The linear correlation coefficient for the whole data set is 0.64 . Finally, we also present the connection between infrared and $\gamma$-ray fluxes and luminosities in Figure 20 that is again in good agreement with our previous results (D’Abrusco et al. 2012; D’Abrusco et al. 2013). 
2FGL J2031.0+1938
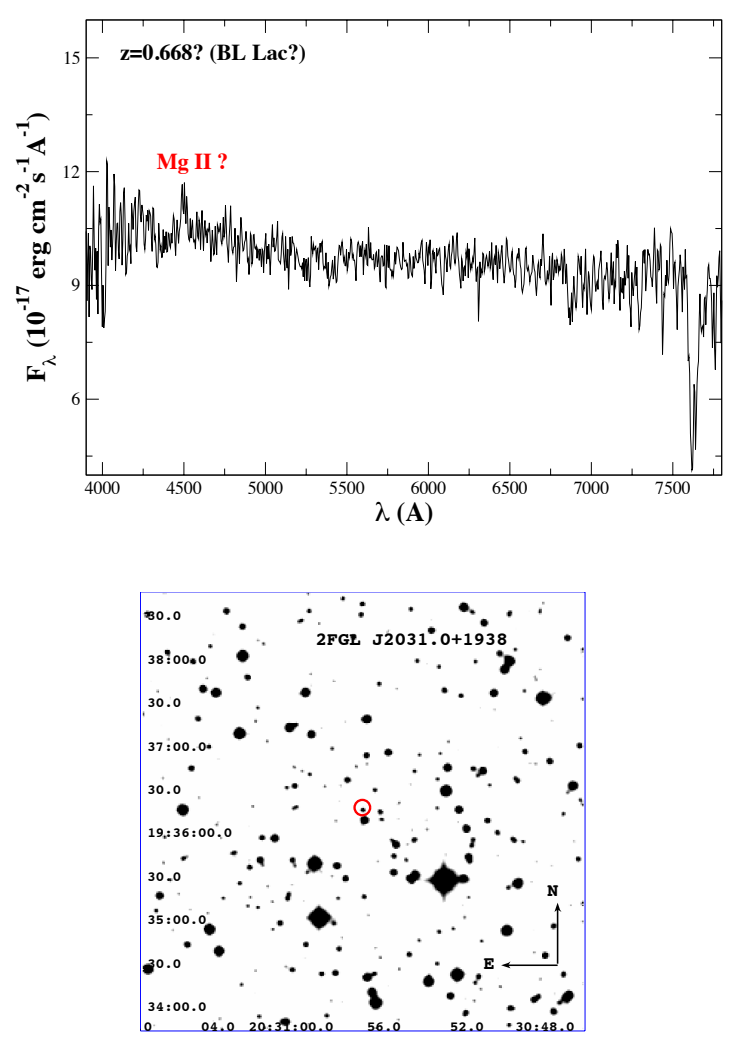

FIG. 14.- Upper panel) The optical spectra of the counterpart associated with 2FGL J2031.0+1938 observed at OAN in San Pedro Mártir (México) on 1 July 2014. The emission line potentially identified as $\mathrm{Mg}_{\mathrm{I}}$ is marked. The source has been classified as a BL Lac. Lower panel) The $5^{\prime} \times 5^{\prime}$ finding chart from the Digitized Sky Survey (red filter). The potential counterpart of 2FGL J2031.0+1938 is indicated by the red circle.

\section{SUMMARY AND CONCLUSIONS}

Two years after the release of the second Fermi source catalog we present a comprehensive multifrequency investigation of all the $\gamma$-ray associations listed in both the 1FGL and the 2FGL catalogs.

First, we introduced a new category of $\gamma$-ray source associations in addition to identified and associated sources. We label as candidate associations those $\gamma$-ray sources having a potential low-energy counterpart of few specific classes of well-known $\gamma$-ray emitters lying within the Fermi positional uncertainty region and/or with angular separations between the Fermi and the counterpart position smaller than the maximum one for all the associated sources of the same class. Then we provided a new classification scheme for the lowenergy counterparts of the Fermi sources based on the multifrequency observations and on the optical spectroscopic information now available. We also presented here a crossmatching between the Fermi catalogs and several surveys of SFRs (see Section 5 for details) to highlight the possibility that an unknown SNR, PWN and/or PSR is embedded therein, and to provide information that could be used to refine models of diffuse Galactic $\gamma$-ray for future releases of the Fermi catalogs.

The total number of $\gamma$-ray sources considered in our investigation for both the 1FGL and the 2FGL comprises 2219 unique Fermi objects, all listed in Table 4 with each assigned
1FGL J2300.4+3138
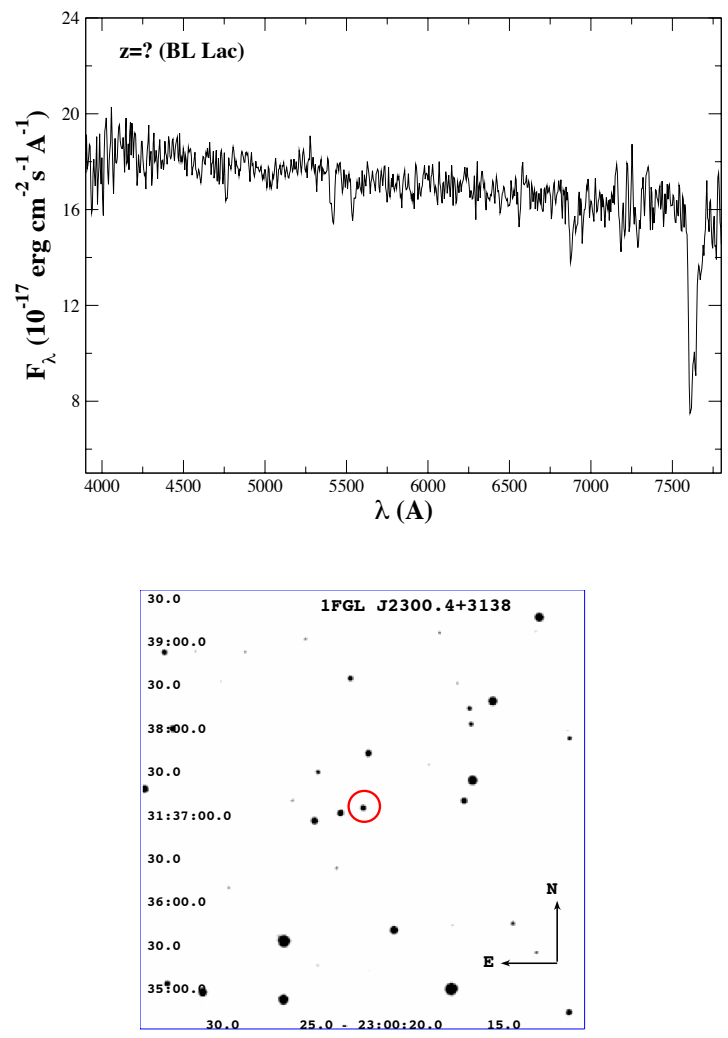

FIG. 15.- Upper panel) The optical spectra of the counterparts associated with 2FGL J2300.4+3138 observed at OAN in San Pedro Mártir (México) on 1 July 2014. Several unidentified absorption features superimposed to the optical continuum are visible; however, we were not able to identify the Civfeature previously reported by Shaw et al. (2013a). (Lower panel) The $5^{\prime} \times 5^{\prime}$ finding chart from the Digitized Sky Survey (red filter). The potential counterpart of 1FGL J2300.4+3138 pointed during our observations is indicated by the red circle.

counterpart and their main multifrequency properties. Overall, in the refined association list of the Fermi catalogs, we found 174 Fermi sources with a SFR consistent with their $\gamma$ ray positions. In particular, 60 Fermi objects out of these 174 do not have the "c" flag in at least one of the Fermi names and include: 13 identified sources, 17 associated, and 30 candidate associations. Their counterparts are also classified as four HMBs; 17 PSRs, among which three lie in a PWN and two in a SNR; three PWNe, one with a PSR; one radio galaxy; thirteen SNRs, two with a PSR included; one binary star; three unclassified sources; and 19 SFRs.

We found spectroscopic information for 177 extragalactic $\gamma$-ray sources not reported in the previous version of the Fermi catalogs. We included analyses of eight new optical spectroscopic observations performed with the 2.1-meter telescope of the OAN in San Pedro Mártir and with the OAGH to confirm the natures of these blazar-like sources found in our multifrequency investigation. Blazar candidates and counterparts with unknown origin (i.e., unc) were also analyzed with the $\mathrm{KDE}$ technique to determine the fraction having WISE IR colors similar to known $\gamma$-ray blazars. We compared the refined associations listed in both the Fermi catalogs with the classification proposed on the basis of statistical investigations (Ackermann et al. 2012; Mirabal et al. 2012) and we found a 
1FGL J2341.6+8015
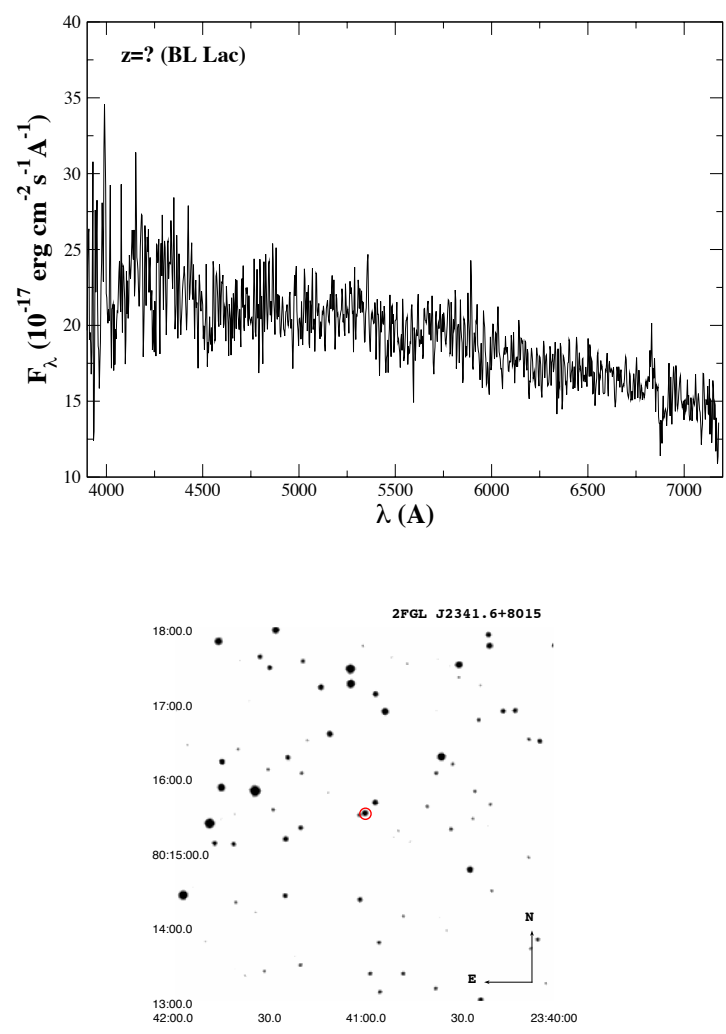

FIG. 16.- Upper panel) The optical spectra of the counterparts associated with 1FGL J2341.6+8015 observed at OAGH on 28 August 2014. The source has been classified as a BL Lac on the basis of its featureless continuum. Lower panel) The $5^{\prime} \times 5^{\prime}$ finding chart from the Digitized Sky Survey (red filter). The potential counterpart of 1FGL J2341.6+8015 is indicated by the red circle.

good agreement with our results.

We note that in the combined list of refined associations for 1FGL and 2FGL, among 2219 sources, 394 are still UGSs (i.e., $\sim 18 \%$ of the entire sample) with 191 of them having no $\gamma$-ray analysis flags. This clean sample of 191 UGSs appears to have a uniform distribution in the sky with a small excess toward the Galactic plane, as shown in Figure 21. Moreover, we conclude that the fraction of Fermi sources with plausible counterparts, combining identifications, associations and candidate associations, within their $\gamma$-ray positional uncertainty regions, is $\sim 80 \%$ and up to $\sim 90 \%$ when considering sources with no $\gamma$-ray analysis flags.

Finally, we remark that within the sample of 191 UGSs that do not have any $\gamma$-ray analysis flags are 40 sources (listed in Table 11) that do not have any NVSS and/or SUMSS radio source within their positional uncertainty regions at $95 \%$ level of confidence. Their all-sky distribution, separated into those lying in the NVSS footprint (i.e., declination greater than -40 $\left.{ }^{\circ}\right)$ and the others observable from the Southern hemisphere is shown in Figure 22. The excess in the subsample of those with declinations less than $-40^{\circ}$, clearly visible in Figure 22, could be due to shallower coverage by SUMSS relative to the NVSS catalog. It is worth mentioning that for these 40 UGSs the distributions of the $\gamma$-ray spectral index and of the $\gamma$-ray fluxes do not show any significant differences from those of BZQs and the BZBs (see Figure 23). However it is intriguing
Radio - $\gamma$-ray flux densities: Northern sample

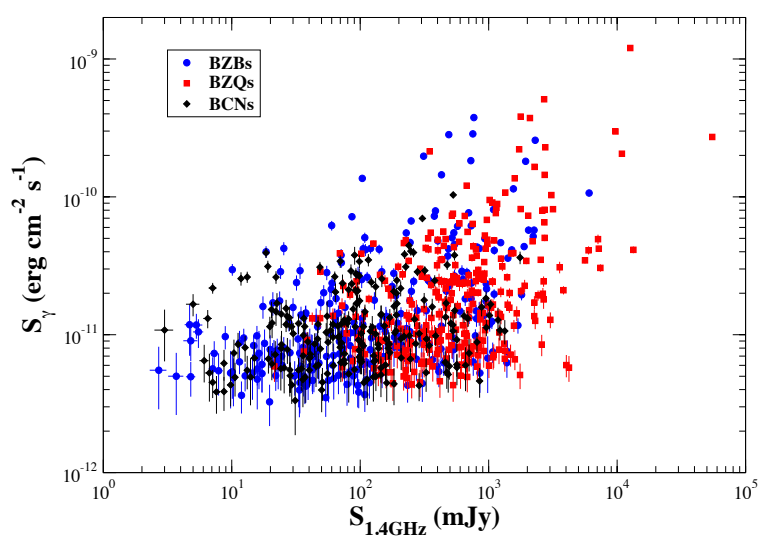

Radio - $\gamma$-ray flux densities: Southern sample

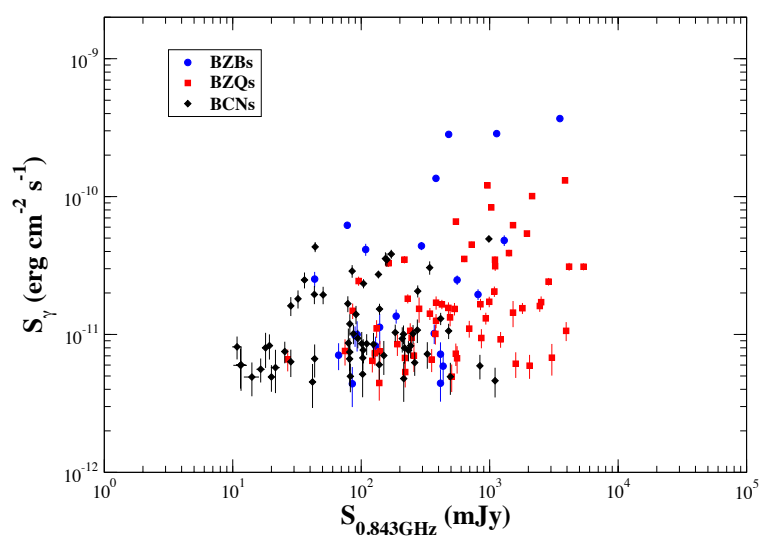

FIG. 17.- Scatter plot of the radio flux densities vs. the $\gamma$-ray flux for blazarlike sources. Spectroscopically confirmed BZBs and BZQs are shown in blue circles and red squares, respectively, while ben sources are black diamonds. Sources with a radio counterpart in the NVSS are in the upper panel while the sources in SUMSS are in the lower panel.

that these UGSs tend to be brighter than the BZB population in the gamma-rays. It is unlikely that this small UGS subsample is constituted by blazars since given their $\gamma$-ray fluxes $S_{\gamma}$ and assuming the typical radio- $\gamma$-ray fluxes of the blazar population, the expected radio flux densities should be greater than $\sim 50 \mathrm{mJy}$ for a large fraction, well above the NVSS and the SUMSS flux thresholds. On the other hand we also conclude that their steep values of $\gamma$-ray spectral indices are not compatible with sources emitting via dark matter annihilation (e.g., Belikov et al. 2012; Drlica-Wagner et al. 2014).

We thank the anonymous referee for useful comments that led to improvements in the paper. F. Massaro and G. Tosti also thank L. Costamante. This investigation is supported by the NASA grants NNX12AO97G and NNX13AP20G. The work by G. Tosti is supported by the ASI/INAF contract I/005/12/0. H. A. Smith acknowledges partial support from NASA/JPL grant RSA 1369566 . HOF was funded by a postdoctoral UNAM grant and is currently granted by a Cátedra CONACyT para Jóvenes Investigadores. V. Chavushyan acknowledges funding by CONACyT research grant 151494 (México). We thank the staff at the Observatorio Astronómico 
Radio - $\gamma$-ray luminosities: Northern sample

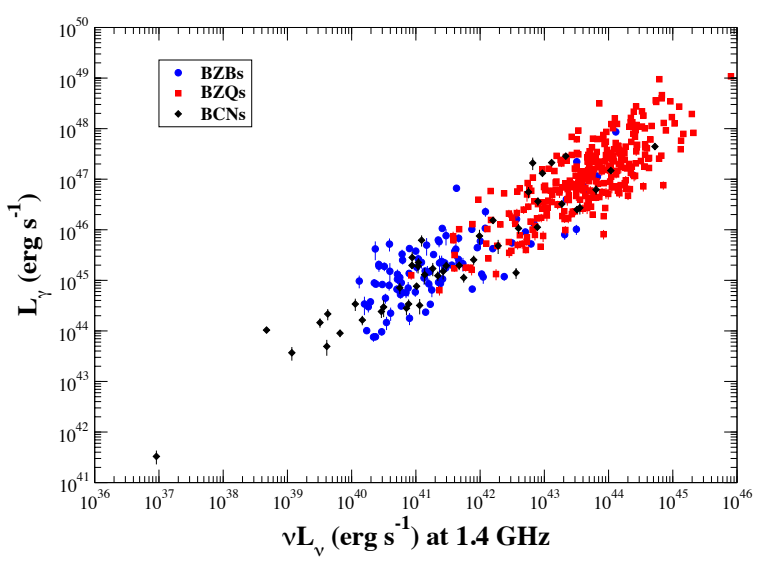

Radio - $\gamma$-ray luminosities: Southern sample

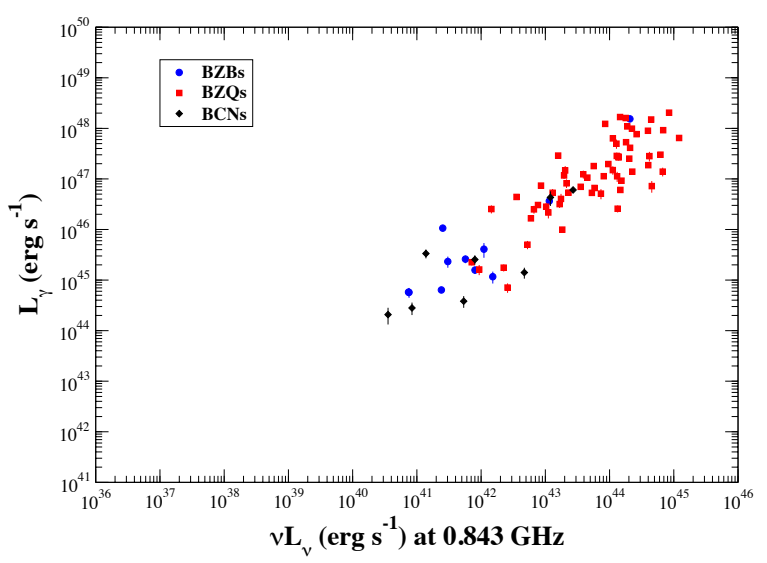

FIG. 18. - Scatter plot of the radio - $\gamma$-ray luminosities for blazar-like sources. Spectroscopically confirmed BZBs and BZQs are shown in blue circles and red squares, respectively, while bcns sources are black diamonds. Sources with a radio counterpart in the NVSS are in the upper panel while the sources in SUMSS are in the lower panel.

Nacional in San Pedro Mártir (México) for all their help during the observation runs. Part of this work is based on archival data, software or on-line services provided by the ASI Science Data Center. This research has made use of data obtained from the high-energy Astrophysics Science Archive Research Center (HEASARC) provided by NASA's Goddard Space Flight Center; the SIMBAD database operated at CDS, Strasbourg, France; the NASA/IPAC Extragalactic Database (NED) operated by the Jet Propulsion Laboratory, California Institute of Technology, under contract with the National Aeronautics and Space Administration. Part of this work is based on the NVSS (NRAO VLA Sky Survey): The National Radio Astronomy Observatory is operated by Associated Universities, Inc., under contract with the National Science Foundation and on the VLA Low-frequency Sky Survey (VLSS). The Molonglo Observatory site manager, Duncan CampbellWilson, and the staff, Jeff Webb, Michael White and John Barry, are responsible for the smooth operation of Molonglo Observatory Synthesis Telescope (MOST) and the dayto-day observing programme of SUMSS. The SUMSS survey is dedicated to Michael Large whose expertise and vision made the project possible. The MOST is operated by
Infrared - $\gamma$-ray Spectral Indices

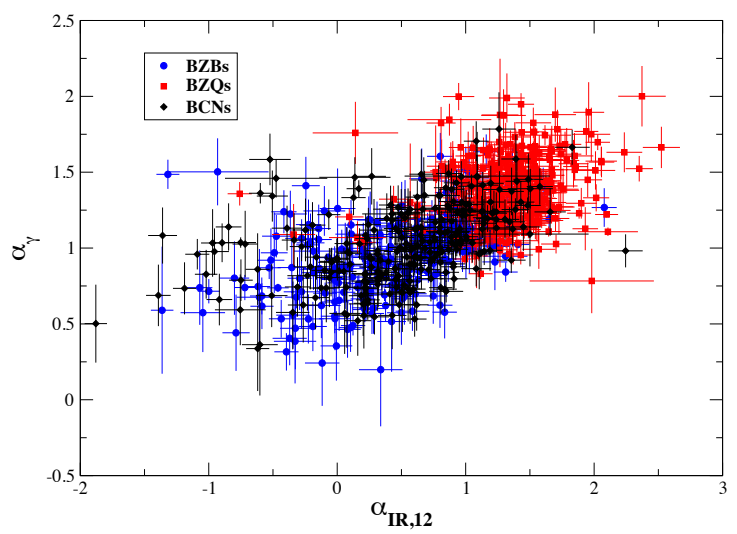

FIG. 19. - Scatter plot of the infrared spectral index evaluated using the first two WISE bands vs. the $\gamma$-ray index for the blazar-like sources in our merged list of refined Fermi associations. Spectroscopically confirmed BZBs and BZQs are shown in blue circles and red squares, respectively, while ben sources are black diamonds.
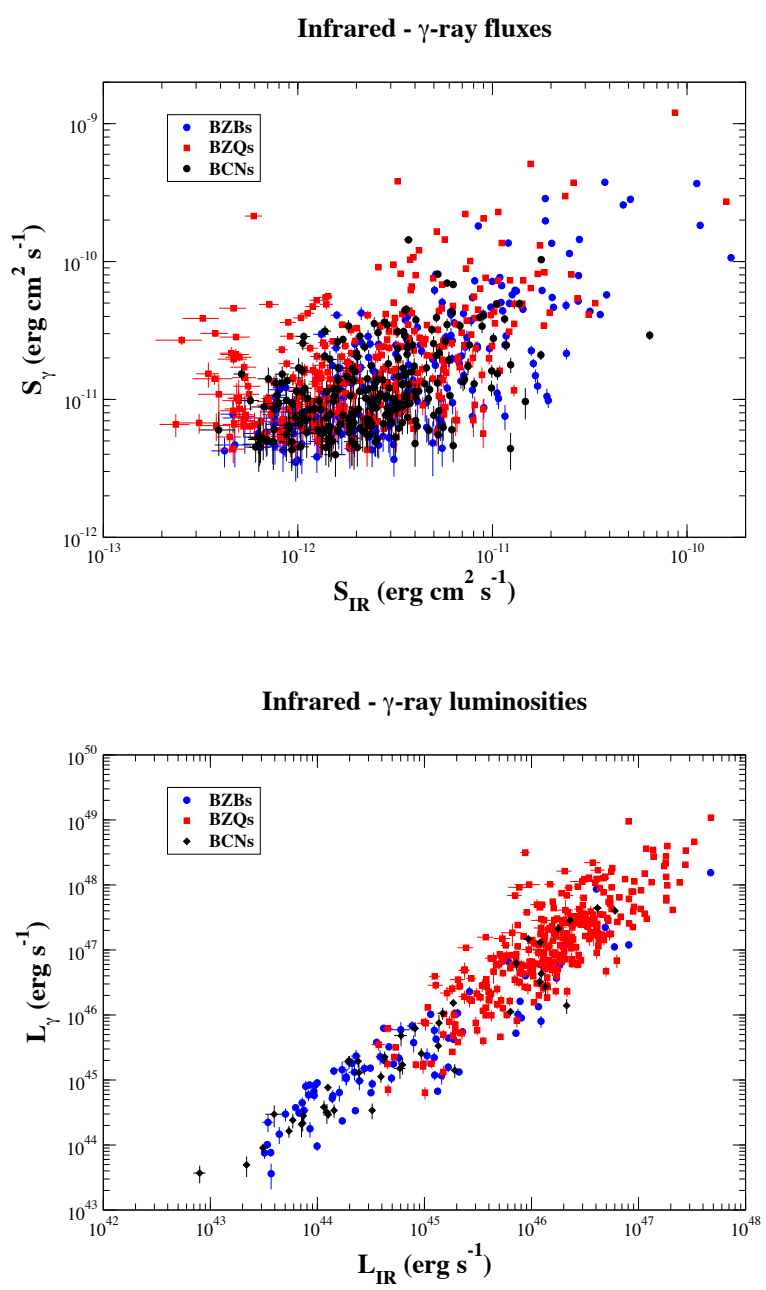

FIG. 20. - The scatterplot of the infrared - $\gamma$-ray fluxes (upper panel) and luminosities (lower panel) for the blazar-like sources. Spectroscopically confirmed BZBs and BZQs are shown in blue circles and red squares, respectively, while ben sources are black diamonds. 


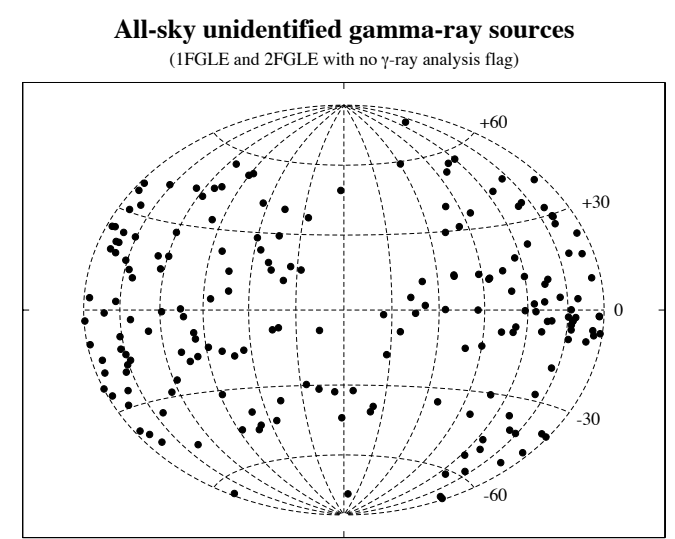

FIG. 21.- All-sky distribution of the remaining UGSs with no $\gamma$-ray analysis flags in the refined association list of the Fermi catalogs.

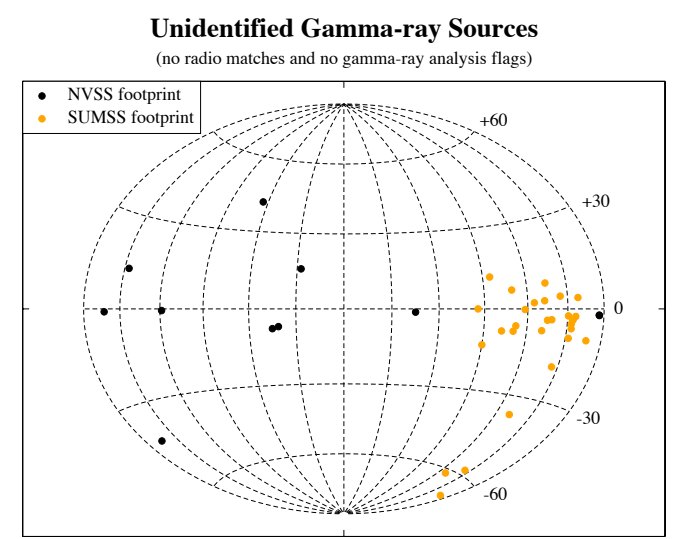

FIG. 22.- All-sky distribution of the 40 UGSs that do not have any NVSS and/or SUMSS radio source within their positional uncertainty regions at 95\% level of confidence and do not present any $\gamma$-ray analysis flag. We show those lying in the NVSS footprint (i.e., declination greater than $-40^{\circ}$ ) as black circles while the others in the Southern hemisphere are indicated as orange circles.

the School of Physics with the support of the Australian Research Council and the Science Foundation for Physics within the University of Sydney. This publication makes use of data products from the Wide-field Infrared Survey Explorer, which is a joint project of the University of California, Los Angeles, and the Jet Propulsion Laboratory/California Institute of Technology, funded by the National Aeronautics and Space Administration. This publication makes use of data products from the Two Micron All Sky Survey, which is a joint project of the University of Massachusetts and the Infrared Processing and Analysis Center/California Institute of Technology, funded by the National Aeronautics and Space Administration and the National Science Foundation. Funding for the SDSS and SDSS-II has been provided by the Alfred P. Sloan Foundation, the Participating Institutions, the National Science Foundation, the U.S. Department of Energy, the National Aeronautics and Space Administration, the Japanese Monbukagakusho, the Max Planck Society, and the Higher
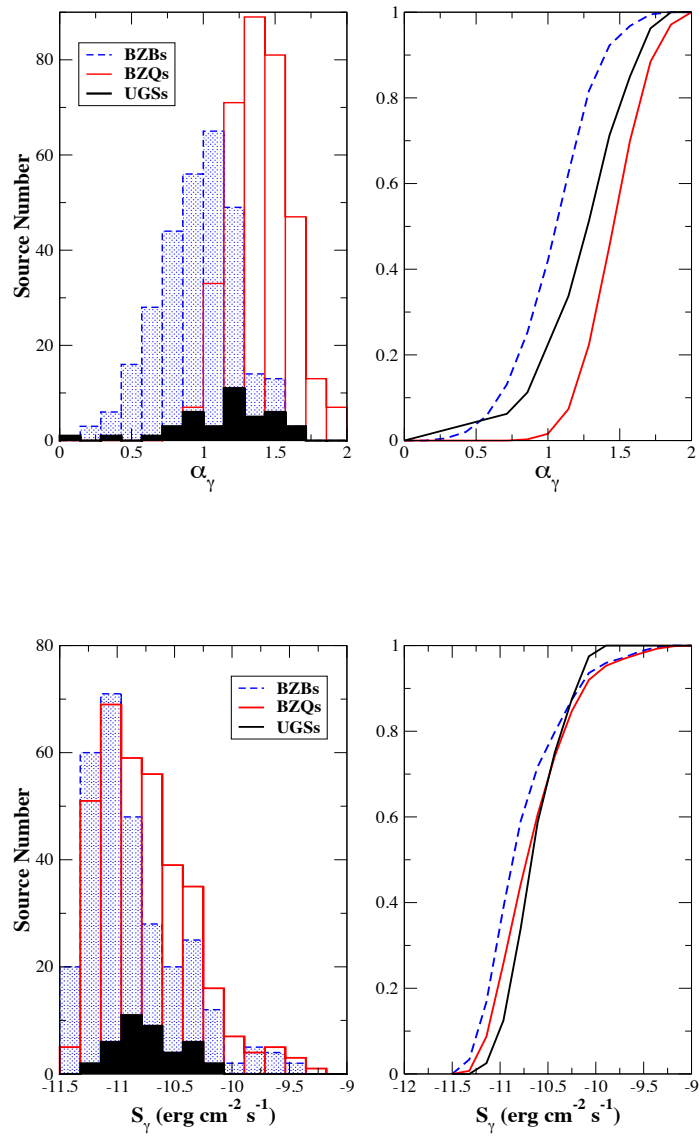

FIG. 23.- The distribution of the $\gamma$-ray spectral index (top) and that of the energy flux (bottom) for the 40 UGSs (straight black line) that do not have any NVSS and/or SUMSS radio source within their positional uncertainty regions and without $\gamma$-ray analysis flags in comparison with those of the BZBs (dashed blue line) and the BZQs (straight red line). Cumulative distributions are also reported on the right panels. The energy flux is the one reported in both the 1FGL and the 2FGL catalogs.

Education Funding Council for England. The SDSS Web Site is http://www.sdss.org/. The SDSS is managed by the Astrophysical Research Consortium for the Participating Institutions. The Participating Institutions are the American Museum of Natural History, Astrophysical Institute Potsdam, University of Basel, University of Cambridge, Case Western Reserve University, University of Chicago, Drexel University, Fermilab, the Institute for Advanced Study, the Japan Participation Group, Johns Hopkins University, the Joint Institute for Nuclear Astrophysics, the Kavli Institute for Particle Astrophysics and Cosmology, the Korean Scientist Group, the Chinese Academy of Sciences (LAMOST), Los Alamos National Laboratory, the Max-Planck-Institute for Astronomy (MPIA), the Max-Planck-Institute for Astrophysics (MPA), New Mexico State University, Ohio State University, University of Pittsburgh, University of Portsmouth, Princeton University, the United States Naval Observatory, and the University of Washington. This research has made use of the USNOFS Image and Catalogue Archive operated by the United States Naval Observatory, Flagstaff Station (http://www.nofs.navy.mil/data/fchpix/). The WENSS project was a collaboration between the Netherlands Foundation for 
TABLE 7

UNIDENTIFIED GaMma-RAY SOURCES WITH NO RADIO MATCHES AND NO $\gamma$-RAY ANALYSIS FLAGS.

\begin{tabular}{|c|c|}
\hline $\begin{array}{l}1 \mathrm{FGL} \\
\text { name }\end{array}$ & $\begin{array}{l}2 \mathrm{FGL} \\
\text { name }\end{array}$ \\
\hline 1FGL J0032.7-5519 & 2FGL J0032.7-5521 \\
\hline 1FGL J0212.3+5319 & 2FGL J0212.1+5318 \\
\hline 1FGL J0545.6+6022 & 2FGL J0545.6+6018 \\
\hline \multicolumn{2}{|l|}{ 1FGL J0709.0-1116 } \\
\hline 1FGL J0802.4-5622 & 2FGL J0802.7-5615 \\
\hline 1FGL J0854.6-4504 & 2FGL J0854.7-4501 \\
\hline 1FGL J0933.9-6228 & 2FGL J0934.0-6231 \\
\hline 1FGL J1036.2-6719 & 2FGL J1036.1-6722 \\
\hline 1FGL J1117.0-5339 & 2FGL J1117.2-5341 \\
\hline 1FGL J1306.4-6038 & 2FGL J1306.2-6044 \\
\hline \multicolumn{2}{|l|}{ 1FGL J1405.5-5846 } \\
\hline \multicolumn{2}{|l|}{ 1FGL J1441.8-6404 } \\
\hline \multirow{2}{*}{\multicolumn{2}{|c|}{$\begin{array}{l}\text { 1FGL J1518.0-5233 } \\
\text { 1FGL J1639.5-5152 }\end{array}$}} \\
\hline & \\
\hline \multicolumn{2}{|l|}{ 1FGL J1650.3-5410 } \\
\hline \multirow{2}{*}{\multicolumn{2}{|c|}{$\begin{array}{l}\text { 1FGL J1702.4-5653 } \\
\text { 1FGL J1743.5-3314 }\end{array}$}} \\
\hline & \\
\hline \multicolumn{2}{|l|}{ 1FGL J1806.2+0609 } \\
\hline \multicolumn{2}{|l|}{ 1FGL J1825.7-1410c } \\
\hline \multicolumn{2}{|l|}{ 1FGL J1948.6+2437 } \\
\hline 1FGL J2112.5-3044 & 2FGL J21 12.5-3042 \\
\hline \multicolumn{2}{|l|}{ 1FGL J2227.4-7804 } \\
\hline \multirow{2}{*}{\multicolumn{2}{|c|}{ 1FGL J2325.8-4043 }} \\
\hline & 2FGL J2332.5-5535 \\
\hline & 2 FGL J0237.9+5238 \\
\hline & 2FGL J1016.4-4244 \\
\hline & 2FGL J1058.7-6621 \\
\hline & 2FGL J1208.5-6240 \\
\hline & 2FGL J1404.0-5244 \\
\hline & 2FGL J1410.4+7411 \\
\hline & 2FGL J1422.3-6841 \\
\hline & 2FGL J1507.0-6223 \\
\hline & 2FGL J1617.3-5336 \\
\hline & 2FGL J1626.4-4408 \\
\hline & 2FGL J1639.8-5145 \\
\hline & 2FGL J1641.8-5319 \\
\hline & 2FGL J1643.3-4928 \\
\hline & 2FGL J1741.1-6750 \\
\hline & 2FGL J1742.5-3323 \\
\hline & 2FGL J1753.8-4446 \\
\hline
\end{tabular}

Research in Astronomy and the Leiden Observatory. We acknowledge the WENSS team consisted of Ger de Bruyn, Yuan Tang, Roeland Rengelink, George Miley, Huub Rottgering, Malcolm Bremer, Martin Bremer, Wim Brouw, Ernst Raimond and David Fullagar for the extensive work aimed at producing the WENSS catalog. TOPCAT ${ }^{30}$ (Taylor 2005) for the preparation and manipulation of the tabular data and the images. The Aladin Java applet ${ }^{31}$ was used to create the finding charts reported in this paper (Bonnarell et al. 2000). It can be started from the CDS (Strasbourg - France), from the CFA (Harvard - USA), from the ADAC (Tokyo - Japan), from the IUCAA (Pune - India), from the UKADC (Cambridge - UK), or from the CADC (Victoria - Canada).

\section{REFERENCES}

Abdo, A. A., Ackermann, M., Ajello, M. et al. 2009 PhRvL, 103, 1101 Abdo, A. A., Ackermann, M., Ajello, M. et al. 2010a ApJS, 188, 405 Abdo, A. A., Ackermann, M., Ajello, M. et al. 2010b ApJ, 715, 429 Abdo, A. A., Ackermann, M., Ajello, M. et al. 2010c ApJ, 723, 649 Abdo, A. A., Ackermann, M., Ajello, M. et al. 2010d A\&A, 523, 2 Abdo, A. A., Ajello, M., Allafort, A. et al. 2013 ApJS, 208, 17 Acero, F., Donato, D., Ojha, R. et al. 2013 ApJ, 779, 133

Ackermann, M., Ajello, M., Allafort, A. et al. 2011a ApJ, 743, 171 Ackermann, M., Ajello, M., Allafort, A. et al. 2011b ApJ, 741, 30 Ackermann, M., Ajello, M., Allafort, A. et al. 2012 ApJ, 753, 83 Ackermann, M., Ajello, M., Allafort, A. et al. 2013 ApJ, 209, 34 Ahn, C. P., Alexandroff, R., Allende Prieto, C. et al. 2012, ApJS, 203, 21 Anderson, L. D., Bania, T. M., Balser, Dana S. et al. 2014 ApJS, 212, 1 Araudo A., et al. 2007, A\&A, 476, 1289

Atwood, W. B., Abdo, A. A., Ackermann, M. et al. 2009 ApJ, 697, 1071

Bade, N., Fink, H. H.,Engels, D. et al. 1995 A\&AS, 110, 469

Bauer, F. E., Condon, J. J., Thuan, T. X. et al. 2000 ApJS, 129, 547

Baker, J. C., Hunstead R. W. \& Brinkmann W. 1995 MNRAS, 277, 553

Becker, R. H., White, R. L., Helfand, D. J., Zoonematkermani, S. 1994 ApJS, 347, 92

Becker, R. H., White, R. L., Helfand, D. J. 1995 ApJ, 450, 559

Belikov, A. V., Buckley, M. R., Hooper, D. 2012 PhRvD, 86d3504

Bennett, A. S. 1962 MNRAS, 125, 75

Berlin, A. \& Hooper, D. 2014 PhRvD, 89a, 6014

Beuermann K., Thomas H.-C., Reinsch K. et al. 1999 A\&A, 347, 47

Bica, E.; Dutra, C. M.; Soares, J.; Barbuy, B. 2003 A\&A, 404, 223

Bikmaev, I. F., Burenin, R. A., Revnivtsev, M. G. et al. 2008 AstL, 34, 653

Bosch-Ramon V., et al., 2010 A\&A 511, A8

\footnotetext{
${ }^{30} \mathrm{http}: / /$ www.star.bris.ac.uk/ mbt/topcat/

31 http://aladin.u-strasbg.fr/aladin.gml
}

Bonnarel, F., Fernique, P., Bienaymé, O. et al. 2000 A\&AS, 143, 33 Britzen, S., Brinkmann, W., Campbell, R. M. et al. 2007 A\&A, 476, 759 Cheung, C. C.; Donato, D.; Gehrels, N.; Sokolovsky, K. V.; Giroletti, M. 2012 ApJ, 756, 33

Cheung, C. C., Shore, S. N., Jean, P. Proceedings of the AAS Meeting \#223, $\# 113.01$

Codella, C., Felli, M., Natale, V. 1994 A\&A, 284, 233

Cohen, A. S., Lane, W. M., Cotton, W. D. et al. 2007 AJ, 134, 1245

Condon, J. J., Cotton, W. D., Greisen, E. W. et al. 1998, AJ, 115, 1693

Cowperthwaite, Philip S. et al. 2013 AJ, 146, 110

Cutri et al. 2012 wise.rept, 1C

Culverhouse, T., Ade, P., Bock, J. et al. 2011 ApJS, 195, 8

D’Abrusco, R., Longo, G., Walton, N. A. 2009 MNRAS , 396, 223

D'Abrusco, R., Massaro, F., Ajello, M., Grindlay, J. E., Smith, Howard A. \& Tosti, G. 2012 ApJ, 748, 68

D’Abrusco, R., Massaro, F., Paggi, A. et al. 2013 ApJS, 206, 12

De Breuck, C., Tang, Y., de Bruyn, A. G. et al. 2002 A\&A, 394, 59

de Ruiter, H. R., Willis, A. G., Arp, H. C. 1977 A\&AS, 28, 211

Del Valle, M. V. \& Romero, G. E. 2012 A\&A 543, A56

Doert, M. \& Errando, M. 2014 ApJ, 782, 41

Douglas, J. N., Bash, F. N., Bozyan, F. A., Torrence, G. W., Wolfe, C. 1996 AJ, 111, 1945

Draine, B. T. 2003, ARA\&A, 41, 241

Drlica-Wagner, A.; Gomez-Vargas, G. A.; Hewitt, J. W., Linden, T., Tibaldo, L.

Drinkwater, M. J., Webster, R. L., Francis, P. J. et al. 1997 MNRAS, 284, 85 Edge, D.O., Shakeshaft, J. R., McAdam, W. B., Baldwin, J. E., Archer, S. 1959 MmRAS, 68, 37

Elvis, M., Maccacaro, T., Wilson, A. S. et al. 1978, MNRAS, 183, 129 Espinoza, C. M. et al. 2013 MNRAS, 430, 571

Evans, I. N., Primini, F. A., Glotfelty, K. J. et al. 2010 ApJS, 189, 37

Evans, P. A., Osborne, J. P., Beardmore, A. P. et al. 2014 ApJS, 210, 8 
Ferrand, G., Safi-Harb, S. 2012 AdSpR, 49, 9, 1313

Ghirlanda, G., Ghisellini, G., Tavecchio, F. \& Foschini, L. 2010, MNRAS 407, 791

Ghirlanda, G., Ghisellini, G., Tavecchio, F., Foschini, L. \& Bonnoli, G. 2011, MNRAS, 413, 852

Giommi, P. et al. 2012 A\&A, 541A.160

Giveon, U., Becker, R. H., Helfand, D. J. et al. 2005 AJ, 129, 348

Green, A. J., Cram, L. E., Large, M. I., Ye, Taisheng 1999 ApJS, 122, 207

Green D. A., 2009, Bulletin of the Astronomical Society of India, 37, 45

Gregory, P. C. \& Condon, J. J. 1991 ApJS, 75, 1011

Gregory, P. C., Scott, W. K., Douglas, K., Condon, J. J. 1996 ApJS, 103, 427

Hanabata Y. et al. 2014 ApJ, 786, 145

Hassan, T., Mirabal, N., Contreras, J. L., Oya, I. 2013 MNRAS, 428, 220

Healey, S. E., Romani, R. W., Taylor, G. B. et al. 2007 ApJS, 171, 61

Healey, S. E.; Romani, R. W., Cotter, G. et al. 2008 ApJS, 175, 97

Hewett, P. C. \& Wild, V. 2010 MNRAS, 405, 2302

Hewitt, A., Burbidge, G. 1989 QSO, M, 0000

Hobbs G. et al. 2004 MNRAS, 352, 1439

Hovatta, T., Lister, M. L., Aller, M. F. et al. 2012 AJ, 144, 105

Hovatta, T., Aller, M. F., Aller, H. D. et al. 2014 AJ, 147, 143

Hughes, V. A. \& MacLeod 1989 AJ, 97, 786

Kataoka, J.; Yatsu, Y.; Kawai, N. 2012 ApJ, 757, 176

Kovalev, Y. Y. 2009 ApJ, 707L, 56

Kuchar, T. A. \& Clark, F. O. 1997 ApJ, 488, 224

Johnston, K. J., Fey, A. L., Zacharias, N. et al. 1995 AJ, 110, 880

Jones, H. D. et al. 2004 MNRAS, 355, 747

Jones, H. D. et al. 2009 MNRAS, 399, 683

Landoni, M. Massaro, F. Paggi, A. et al. 2014 AJ submitted

Landt H., Padovani, P., Eric S. P. et al. 2001 MNRAS, 323, 757

Lane, W. M., Cotton, W. D., van Velzen, S. et al. 2014 MNRAS, 440, 327

Laurino, O., D’Abrusco, R., Longo, G., Riccio, G. 2011 MNRAS, 418, 2165

Lister, M. L., Aller, M., Aller, H. et al. 2011 ApJ, 742, 27

López-Caniego, M. et al. 2013 MNRAS, 430, 1566

Maeda, K.; Kataoka, J.; Nakamori, T. et al. 2011 ApJ, 729, 103

Mahony, E. K., Sadler, E. M., Murphy, T., et al. 2010, ApJ, 718, 587

Manchester, R. N., Hobbs, G.B., Teoh, A. \& Hobbs, M. 2005, AJ, 129, 1993

Mao, L. S. 2011 NewA, 16, 503

Marti, J., Paredes, J. M., Bloom, J. S. et al. 2004 A\&A, 413, 309

Maselli, A., Cusumano, G., Massaro, E. et al. 2010 A\&A, 520A, 47

Masetti, N., Sbarufatti, B., Parisi, P. 2013 A\&A, 559A, 58

Massaro, E., Giommi, P., Leto, C. et al. 2009 A\&A, 495, 691

Massaro, F., D’Abrusco, R., Ajello, M., Grindlay, J. E. \& Smith, H. A. 2011a ApJ, 740L, 48

Massaro, E., Giommi, P., Leto, C. et al. 2011b "Multifrequency Catalogue of Blazars (3rd Edition)", ARACNE Editrice, Rome, Italy

Massaro, F., D’Abrusco, R., Tosti, G., Ajello, M., Gasparrini, D., Grindlay, J. E. \& Smith, Howard A. 2012a ApJ, 750, 138

Massaro, F., D’Abrusco, R., Tosti, G., Ajello, M., Paggi, A., Gasparrini, 2012b ApJ, 752, 61

Massaro, F., D’Abrusco, R., Paggi, A., Tosti, G., Gasparrini, D. 2012c ApJ, 750L, 35

Massaro, F., D’Abrusco, R., Paggi, A. et al. 2013a ApJS, 206, 13

Massaro, F., D’Abrusco, R., Giroletti, M. et al. 2013b ApJS, 207, 4

Massaro, F., D'Abrusco, R., Paggi, A. et al. 2013c ApJS, 209, 10

Massaro, F., Giroletti, M., Paggi, A. et al. 2013d ApJS, 208, 15

Massaro, F., Giroletti, M., D’Abrusco, R. et al. 2014a ApJS, 213, 3

Massaro, F., Masetti, N., D’Abrusco, R. et al. 2014b AJ in press

Massaro, F., Masetti, N., D’Abrusco, R. et al. 2014c A\&A submitted

Mattox, J. R., Schachter, J., Molnar, L., Hartman, R. C., Patnaik, A. R. 1997 ApJ, 481, 95

Mattox, J. R., Hartman, R. C., Reimer, O. 2001 ApJS, 135, 155

Matheson, T. et al., 2008, AJ, 135, 1598

Mauch, T., Murphy, T., Buttery, H. J. et al. 2003 MNRAS, 342, 1117

Maza, J., Wischnjewsky, M., Antezana, R. \& González, L. E. 1995 RMxAA, 31,119

Mirabal, N. 2009 ApJ, 701, 129

Mirabal, N.; Frías-Martinez, V.; Hassan, T.; Frías-Martinez, E. 2012

MNRAS, 424L, 64

Mirabal, N. 2013a MNRAS, 432L, 71

Mirabal, N. 2013b MNRAS, 436, 2461

Mitton, S., Hazard, C., and Whelan, J. J. 1977 MNRAS, 179, 569

Monet, D. G. et al. 2003 AJ, 125, 984

Montmerle, T. 1979 ApJ, 231, 95

Montmerle, T. 2009 Conf. "High-Energy Phenomena in Massive Stars",

Jaen (Spain), Feb. 2-5, 2009, 14 p. [arxiv.org/abs/0909.0222]

Murphy, T. et al. 2010 MNRAS, 402, 2403

Moskalenko, I. V., Digel, S. W., Porter, T. A. et al. 2007 NuPhS, 173, 44
Mucke, A., Pohl, M., Reich, P., et al. 1997, A\&A, 320, 33

Muller, C., Kadler, M., Ojha, R. et al. 2014 A\&A, 562A, 4

Nolan, P. L., Abdo, A. A., Ackermann, M. et al. 2012 ApJS, 199, 31

Nori, M., Giroletti, M., Massaro, F., D’Abrusco, R., Paggi, A. \& Tosti, G. et al. 2014 ApJS 212, 3

Padovani, P., Ghisellini, G., Fabian, A. C. \& Celotti, A. 1993, MNRAS, 260, L21

Paggi, A., Massaro, F., D’Abrusco, R. et al. 2013 ApJS, 209, 9

Paggi, A., Milisavljevic, D., Masetti, N. et al. 2014 AJ, 147, 112

Paladini, R., Burigana, C., Davies, R. D. et al. 2003 A\&A 397, 213

Pavlidou, V. et al. 2012 ApJ 751, 149

Planck Collaboration 2014 A\&A submitted [arXiv:1303.0576]

Petrov, L., Phillips, C., Bertarini, A., Murphy, T., Sadler, E. M. 2011

MNRAS, 414, 2528

Petrov, L., Mahony, E. K., Edwards, P. G. et al. 2013 MNRAS, 432, 1294

Pletsch, H. J. et al. 2012a ApJ, 744, 105

Pletsch, H. J. et al. 2012b ApJ, 755L, 20

Pletsch, H. J. et al. 2013 ApJ, 779L, 11

Prestage, R. M., \& Peacock, J. A., 1983, MNRAS, 204, 355

Quintana, H. \& Ramirez A. 1995 ApJS, 96, 343

Raiteri, C. M., Villata, M., Carnerero, M. I. et al. 2014 MNRAS, 442, 629

Ray, P. S. et al. 2012 [arXiv:1205.3089]

Ray, P. S. et al. 2013 ApJ, 763L, 13

Rengelink, R., Tang, Y., de Bruyn, A. G. et al. 1997, A\&A Suppl. 124, 259

Richter, G. A., 1975, Astroh. Nachrichten, 296, 65

Romani, R. W., Filippenko, A. V., Cenko, S. B. 2014 ApJL submitted [arXiv:1408.2886]

Rowell, G. et al. "Proceedings of the 25th Texas Symposium on Relativistic Astrophysics." December 6-10, 2010. Heidelberg, Germany. Editors:

Frank M. Rieger (Chair), Christopher van Eldik and Werner Hofmann.

Salamon, M. H. \& Stecker, F. W. 1994, ApJ, 430, L21

Saxton, R. D. 2008 A\&A, 480, 611

Schinzel, F. K., Petrov, L., Taylor, G. B. ApJS submitted

[http://arxiv.org/abs/1408.6217]

Sharpless, S. 1959 apJS, 4, 275

Shaw, M. S., Romani, R. W.; Cotter, G. et al. 2013 ApJ, 764, 135

Shaw, M. S., Filippenko, A. V., Romani, R. W. et al. 2013 AJ, 146, 127

Schwope, A., Hasinger, G., Lehmann, I. et al. 2000 AN, 321, 1

Skrutskie, M. F. et al. 2006, AJ, 131, 1163

Spinrad, H., Marr, J., Aguilar, L., Djorgovski, S. 1985 PASP, 97, 932

Stephen, J. B.; Bassani, L., Landi, R., Malizia, A., Sguera, V., Bazzano, A., Masetti, N. 2010 MNRAS, 408, 422

Stern, D. \& Assef, R. J. 2013 ApJ, 764L, 30

Stroh, M. C. \& Falcone, A. D. 2013, ApJS, 207, 28

Stecker, F. W., Salamon, M. H. \& Malkan, M. A. 1993, ApJ, 410, L71

Stickel, M. \& Kuehr, H. 1996a A\&AS, 115, 1

Stickel, M. \& Kuehr, H. 1996b A\&AS, 115, 11

Sutherland, W. \& Saunders, W. 1992 MNRAS, 259, 413

Takahashi, Y.; Kataoka, J.; Nakamori, T. et al. 2012 ApJ, 747, 64

Takeuchi, Y., Kataoka, J., Maeda, K. et al. 2013 ApJS, 208, 25

Taylor A.R., Goss W.M., Coleman P.H. et al. 1996 ApJS, 107, 239

Taylor, G. B., Healey, S. E., Helmboldt, J. F., et al. 2007, ApJ, 671, 1355

Taylor, M. B. 2005, ASP Conf. Ser., 347, 29

Thompson, M. A., Hatchell, J., Walsh, A. J. et al. 2006 A\&A, 453, 1003

Thompson, D. J., Djorgovski, S. \& De Carvalho, R. 1990 PASP, 102, 1235

Tsarevsky G., de Freitas Pacheco, J. A., Kardashev, N. et al. 2005 A\&A, 438,949

Titov, O., Jauncey, D. L., Johnston, H. M. et al. 2011 AJ, 142, 165

Tody, D.1986 SPIE, 627, 733

Vandenbroucke, J., Buehler, R., Ajello, M. et al. 2010 ApJ, 718L, 166

Vettolani, G. Cappi, R. Chincarini, G.

Voges, W. et al. 1999 A\&A, 349, 389

Voges, W. et al. 2000 IAUC, 7432R, 1.

Warwick, R. S.; Saxton, R. D.; Read, A. M. 2012 A\&A, 548A, 99

Werner, M.; Reimer, O.; Reimer, A.; Egberts, K. 2013 A\&A, 555A, 102

White, G. L., Jauncey, D. L. Savage, A. et al. 1988 ApJ 327, 561

White, R. L., Becker, R. H. Helfand, D. J., Gregg, M. D. et al. 1997 ApJ,

475,479

Wolstencroft, R. D. et al. 1986, MNRAS, 223, 279

Wright, A. \& Otrupcek, R. 1990 PKS, C, 0

Wright, A. E., Griffith, M. R., Burke, B. F., Ekers, R. D. 1994 ApJS, 91, 111

Wright, E. L., et al. 2010 AJ, 140, 1868

Zechlin, H.-S., Fernandes, M. V., Elsasser, D., Horns, D. 2012 A\&A, 538A, 93 

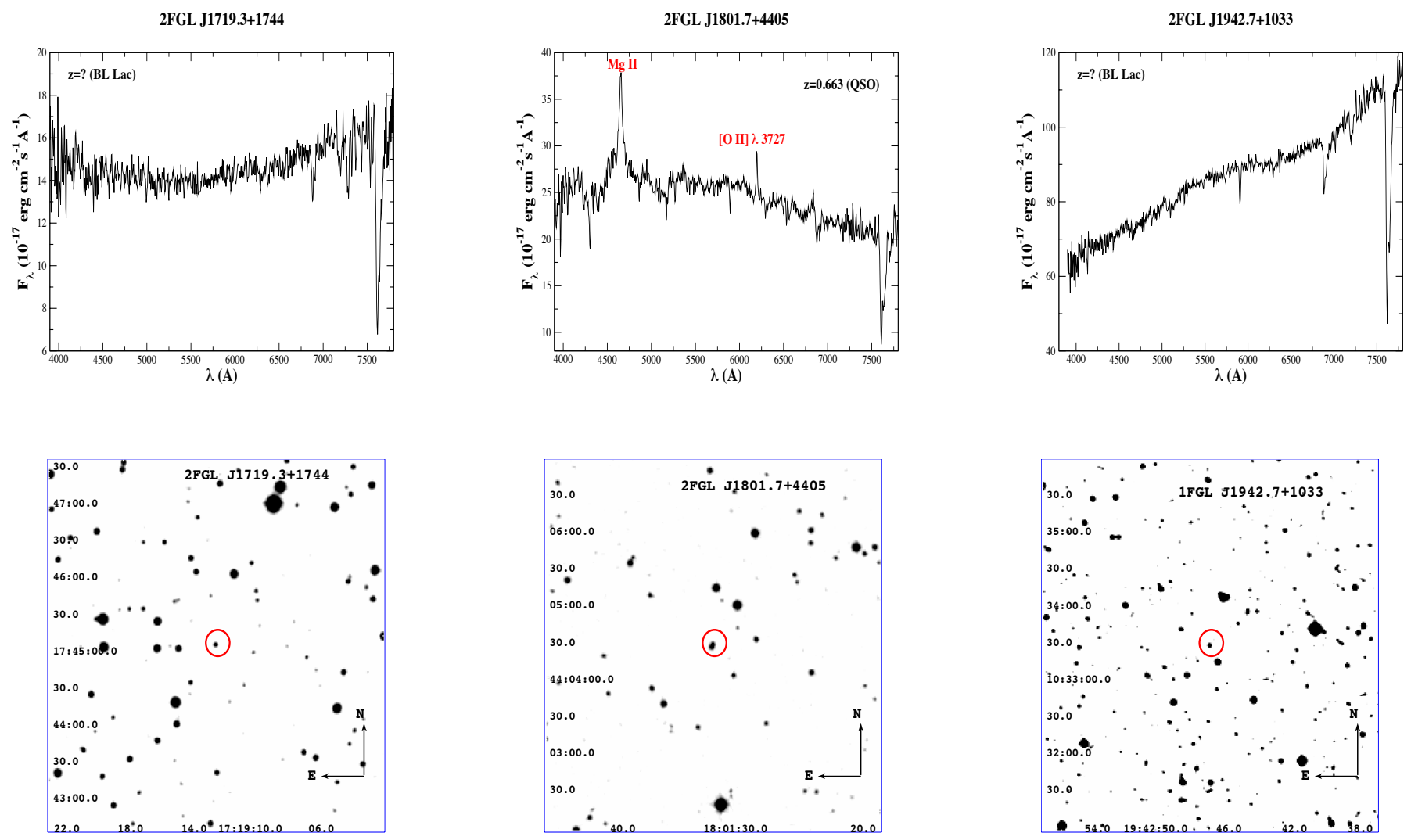

FIG. 24.- Upper panel) The optical spectra of the counterparts associated with 2FGL J1719.3+1744, 2FGL J1801.7+4405 and 1FGL J1942.7+1033 already available in literature and re-observed at OAN in San Pedro Mártir (México) on 29 June and 30 June 2014. The Mg II and the [OII] $\lambda 3727$ emission lines superimposed to the optical continuum are visible in 2FGL J1801.7+4405 and allowed us to classify the source as a quasar, while the lack of features in the other two spectra led toward a BL Lac classification. (Lower panel) The $5^{\prime} \times 5^{\prime}$ finding chart from the Digitized Sky Survey (red filter). The potential counterparts pointed during our observations is indicated by the red circle.

\section{APPENDIX}

Here we present the spectra of 2FGL J1719.3+1744 and 2FGL J1801.7+4405, both with uncertain redshift, as reported in the Roma-BZCAT together with that of 1FGL J1942.7+1033. We confirmed the redshift for 2FGL J1801.7+4405 and the BL Lac classification of 1FGL J1942.7+1033 (Tsarevsky et al. 2005; Masetti et al. 2013) while 2FGL J1719.3+1744 (alias BZBJ1719+1745) has a completely featureless spectrum. 